\title{
Comparative Study of Arithmetic and Huffman Compression Techniques for Enhancing Security and Effective Bandwidth Utilization in the Context of ECC for Text
}

\author{
O.Srinivasa Rao \\ Dept. of CSE, \\ JNTUK University College of Engineering, \\ Vizianagaram, A.P. \\ India-535003
}

\author{
Prof.S.Pallam Setty \\ CS \& SE Dept., \\ Andhra University College of Engineering, \\ Visakapatnam, A.P, \\ India-530003
}

\begin{abstract}
In this paper, we proposed a model for text encryption using elliptic curve cryptography (ECC) for secure transmission of text and by incorporating the Arithmetic/Huffman data compression technique for effective utilization of channel bandwidth and enhancing the security.

In this model, every character of text message is transformed into the elliptic curve points $\left(\mathrm{X}_{\mathrm{m}}, \mathrm{Y}_{\mathrm{m}}\right)$, these elliptic curve points are converted into cipher text .The resulting size of cipher text becomes four times of the original text. For minimizing the channel bandwidth requirements, the encrypted text is compressed using the Arithmetic and Huffman compression technique in the following two ways by considering i) $\mathrm{x}-\mathrm{y}$ coordinates of encrypted text and ii) x-co-ordinates of the encrypted text. The results of the above two cases are compared in terms of overall bandwidth required and saved for Arithmetic and Huffman compression.
\end{abstract}

\section{Keywords}

Elliptic Curve Cryptography (ECC), Text encryption, Huffman compression, Arithmetic compression

\section{INTRODUCTION}

Over last three decades, the traditional cryptosystem like DES, DLP, AES, DSA and RSA etc. are used for privacy and security. But these conventional methods are not able to support the new generation of digital communication and information access devices, these devices required a crypto-security technology. A method called Elliptic Curve Cryptography is becoming the choice for mobile communication. Elliptic curve cipher use very small key size and computationally is very efficient. N. Koblitz [1] and Victor Miller [2], independently proposed the elliptic curve cryptosystem.

One can use an elliptic curve group that is smaller in size while maintaining the same level of security. The result is smaller key sizes, bandwidth savings, and faster implementations - features that are especially attractive for security applications where computational power and integrated circuit space is limited, such as smart cards, personal digital assistants, and wireless devices. Elliptic curve cryptographic protocols for digital signatures, public-key encryption, and key establishment have been standardized by numerous standards organizations including:

- American National Standards Institute (ANSI X9.62 [3], ANSI X9.63 [4])

- Institute of Electrical and Electronics Engineers (IEEE 1363-2000 [5])

- International Standards Organization (ISO/IEC 15946$3[6])$

- U.S. government's National Institute for Standards and Technology (FIPS 186-2 [7])

- Internet Engineering Task Force (IETF PKIX [7], IETF OAKLEY [8])

- $\quad$ Standards for Efficient Cryptography Group (SECG [9])

The vast majority of the products and standards that use publickey cryptography for encryption and digital signatures use RSA [10]. As we have seen, the bit length for secure RSA use has increased over recent years, and this has put a heavier processing load on applications using RSA. This burden has ramifications, especially for electronic commerce sites that conduct large numbers of secure transactions. Recently, a competing system that has emerged is elliptic curve cryptosystem (ECC) [4,11].

\subsection{Elliptic Curve Cryptography}

Elliptic curve cryptography makes use of elliptic curves in which the variables and coefficients are all restricted to elements of a finite field. Two families of elliptic curves are used in cryptographic applications: Prime curves defined over $\mathrm{Z}_{\mathrm{p}}$ and binary curves constructed over GF $\left(2^{\mathrm{m}}\right)$. Fernandez[12] points out that prime curves are best suited for software applications, as the extended bit -fiddling operations needed by binary curves are not required; , and that binary curves are best for hardware applications, where it takes remarkably few logic gates to create a powerful and fast cryptosystem. In this paper we used prime curves defined over $Z_{p}$ for analysis purpose.

\subsection{Mathematical review}

We consider an elliptic curve over prime fields which are of the form:

$E: y^{2}=x^{3}+a x+b \bmod p$ where $a, b \in \mathrm{F} p$ and $4 a^{3}+27 b^{2} \neq 0$ $\bmod p$ 
The addition of two points $P\left(x_{1}, y_{1}\right)$ and $Q\left(x_{2}, y_{2}\right)$ is calculated by:

$$
\begin{gathered}
R\left(x_{3}, y_{3}\right)=P+Q \text { where: } \\
x_{3}=\lambda^{2}-x_{1}-x_{2}, \\
y_{3}=\lambda\left(x_{1}-x_{3}\right)-\mathrm{y}_{1}, \\
\lambda=\left(y_{2}-y_{1}\right) /\left(x_{2}-x_{1}\right) \text { if } P \neq Q \\
\lambda=\left(3 x_{1}^{2}+a\right) / 2 y_{1} \text { if } P=Q
\end{gathered}
$$

\section{DATA COMPRESSION TECHNIQUES}

Compression is a technology for reducing the quantity of data used to represent any content without excessively reducing the quality of the picture. It also reduces the number of bits required to store and/or transmit digital media. Compression is a technique that makes storing easier for large amount of data. The performance of data compression algorithms is measured in terms of compression ratio (CR) which is defined as

\section{Compression ratio $=$ Size of the output stream/size of the input} stream.

We analyzed the adoptability of Huffman data compression techniques for encrypted image data/message in the context of ECC for effective utilization of channel bandwidth for two cases i.e. (i) For different sizes of same image and (ii) For different images of almost same size.

\subsection{Arithmetic Coding Technique for Data Compression}

Due to its high efficiency and the hardware implementation of Arithmetic coding gains more interest. The basic concept of arithmetic coding can be traced back to Elias in the early 1960s (see [13],pp. 61-621). Practical techniques were first introduced by Rissanen [14] and Pasco [15], and developed further by Rissanen [16]. The reader interested in the broader class of arithmetic codes is referred to [17]; a tutorial is available in [18].

Arithmetic coding is superior in most respects to the betterknown Huffman [13] method. In comparison of the Huffman coding algorithm, Arithmetic Coding over comes the constraint that the symbol to be encoded by a whole number of bits. This leads to higher efficiency and a better comparison ratio in general. Indeed Arithmetic coding can be proven to almost reach the best comparison ratio possible, which is bounded by the entropy of the data being encoded. Though during the encoding the algorithm generates one code for the whole input stream, this is done in a fully sequential manner, symbol after symbol.

\section{Huffman Compression Technique}

In 1952, Huffman [13] proposed an elegant sequential algorithm which generates optimal prefix codes in $O(n \log n)$ time. The algorithm actually needs only linear time provided that the frequencies of appearances are sorted in advance [20, 21]. Since then there have been extensive researches on analysis, implementation issues and improvements of the Huffman coding theory in a variety of applications [22, 23, 24, 25, 26, 27 and 28].

Huffman coding, is a particular method of compressing data through the use of a code table with encodings of variable lengths. A Huffman code is an optimum, or minimumredundancy, code, which means that messages which occur with greater probability have shorter encodings; in addition, it is prefix free, meaning that no code in the table may be the beginning part of any other code. Huffman describes an algorithm which can be used to generate a binary Huffman code from a collection of messages, or strings, ordered by probability. To generate a code, one starts with a collection of all messages in order of probability. The two least probable messages are removed from the collection and combined into a "composite message," with probability equal to the sum of the messages comprising it. This process is repeated until there is only a single composite message left in the collection, with a probability of 1 ; that composite message represents the entire Huffman code. This is easily converted to a tree-based approach, in which the initial messages are represented as leaf nodes, each edge represents a digit 0 or 1 in the encoding, and "composite messages" are sub trees created by assigning a common parent to the merged messages.

\section{PROPOSED MODEL FOR ENCRYPTION AND DECRYPTION}

The proposed model at sender and receiver side for text in the context of ECC for enhancing the security and effective utilization of the channel bandwidth is shown in Figure1.The following two sections describes the proposed model at sender side and at receiver side of text encryption and compression technique for secure transmission of the text by aiming the effective utilization of channel bandwidth.

\subsection{Encryption and Compression procedure (at sender side)}

1. Take plain text $X$,

2. Each character of $X$, i.e. assigned as message $P_{m}$, can be converted into the point coordinate $\left(\mathrm{X}_{\mathrm{m}}, \mathrm{Y}_{\mathrm{m}}\right)$ on EC

3. Encryption/decryption system require a point on $G$ and an elliptic group $\mathrm{E}_{\mathrm{p}}(\mathrm{a}, \mathrm{b})$. User $\mathrm{A}$ select a private key $\mathrm{n}_{\mathrm{A}}$ and generate a public key $\mathrm{P}_{\mathrm{A}}=\mathrm{n}_{\mathrm{A}} \times \mathrm{G}$. To encrypt and send pixel $\mathrm{P}_{\mathrm{m}}$, to $\mathrm{B}, \mathrm{A}$ choose a random positive integer $\mathrm{k}$ and produce the cipher text $\mathrm{C}_{\mathrm{m}}$ consisting of the pair of points

$\mathrm{C}_{\mathrm{m}}=\left\{\mathrm{kG}, \mathrm{P}_{\mathrm{m}}+k \mathrm{P}_{\mathrm{B}}\right\}-(1)$, where $\mathrm{P}_{\mathrm{B}}$ is the public key of user $\mathrm{B}$. 4. The $\mathrm{x}$-coordinates/(x, $\mathrm{y})$ coordinates of encrypted cipher text values are compressed by using the Arithmetic/Huffman data compression which is then transmitted through in secure channel to the destination.

\subsection{Proposed Decompression and Decryption (at the receiver side)}

1. Received raw data, i.e. compressed x-coordinates/ (x,y) coordinates of the encrypted text is decompressed using the Arithmetic/Huffman decompression technique

2. To retrieve the cipher text values (if the raw data contains only $x$ coordinates), one need to compute y-coordinates also. These values are generated by substituting the $\mathrm{x}$ co-ordinate values into the chosen elliptic curve

3. To decrypt the cipher pixel, B multiplies the first point in the pair by B's secret key and subtracts the result from the second point:

$$
\mathrm{P}_{\mathrm{m}}+\mathrm{kP}_{\mathrm{B}}-\mathrm{n}_{\mathrm{B}}(\mathrm{kG})=\mathrm{P}_{\mathrm{m}}+\mathrm{k}\left(\mathrm{n}_{\mathrm{B}} \mathrm{G}\right)-\mathrm{n}_{\mathrm{B}}(\mathrm{kG})=\mathrm{P}_{\mathrm{m}}
$$




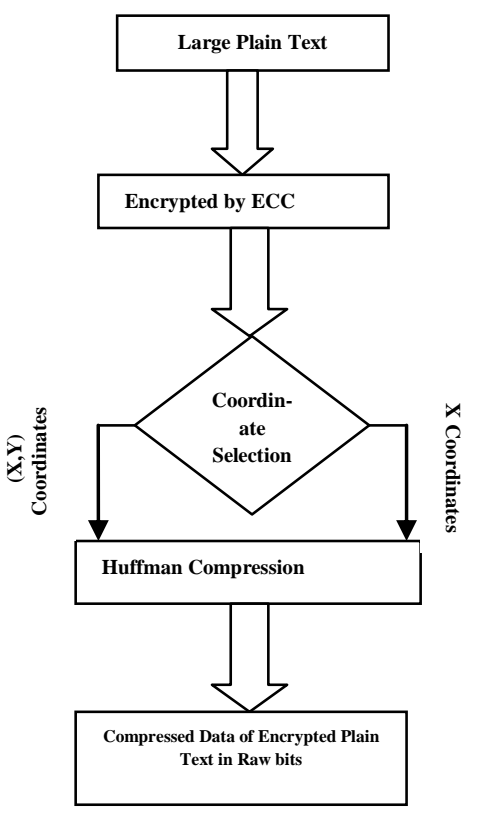

(a) Proposed Encryption \& Compression Procedure (At sender side)

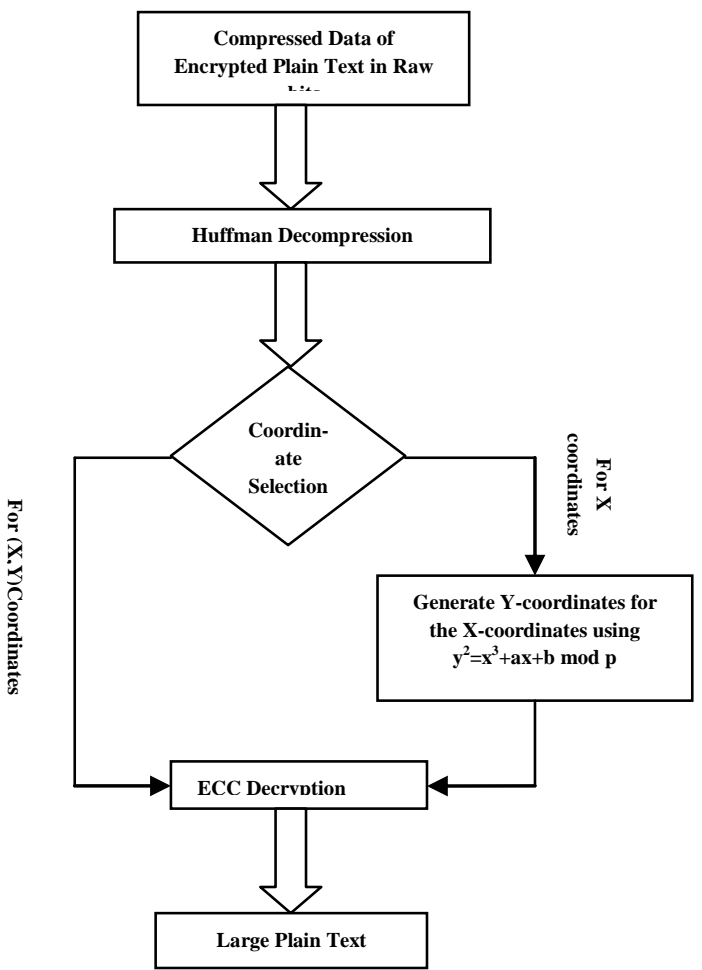

b) Proposed Decompression \& Decryption Procedure (At Receiver side)

\section{Figure 1 Proposed model at sender side and receiver side for images in the context of ECC}

For practical purpose, We have taken an elliptic curve $\mathrm{E}_{571}(1,1)$ in the prime field and the alpha numerical characters are mapped $[29,30]$ to the points of the EC. The mapped points are encrypted [31, 32] and computed compression ratio [33] for encrypted points using Huffman, from which we found the overall percentage of the bandwidth required and the percentage of the bandwidth saved.

The following rules are implemented for reducing the bandwidth:

1. The size of encrypted data size is $n^{*}\left[K G, P_{m}+K P_{B}\right]$ for the $\mathrm{n}$ bytes of the message. If, we send all encrypted data as it is to the destination, then the bandwidth required is $4 *_{n}$ bytes for $n$ byte data message/image, i.e., Four times of the bandwidth required.

2. Instead of sending every point $C_{m}$ we send only once $\mathrm{KG}$ and rest of the $\left[\mathrm{P}_{\mathrm{m}}+\mathrm{KP}_{\mathrm{B}}\right]$ for $\mathrm{n}$ times, i.e., for $4 \mathrm{n}$ bytes of encrypted data we send only $K G+n *\left[P_{m}+\right.$ $\mathrm{KP}_{\mathrm{B}}$ ] bytes to the destination, which is enough to recover the original Message. The amount of bandwidth saved at this stage is:

If the $\mathrm{n}$ value is very large, then, $\mathrm{KG}+\mathrm{n}\left(\mathrm{P}_{\mathrm{m}}+\mathrm{KP}_{\mathrm{B}}\right) \approx$ $n\left(P_{m}+K P_{B}\right)$, hence the reduced bandwidth is give by,

$$
\frac{n\left[P_{m}+K P_{B}\right]}{n\left[K G\left(P_{m}+K P_{B}\right)\right]}=\frac{P_{m}+K P_{B}}{K G\left(P_{m}+K P_{B}\right)}
$$

As we know $\mathrm{KG}$ and $\mathrm{P}_{\mathrm{m}}+\mathrm{KP}_{\mathrm{B}}$ are 1 byte each, so that bandwidth saved to $1 / 2$ of the originally required, i.e., $50 \%$ can be saved at this point.

3. As $\mathrm{n}$ is very large the encrypted data of $\left[\mathrm{KG}, \mathrm{n} *\left(\mathrm{P}_{\mathrm{m}}+\right.\right.$ $\left.\mathrm{KP}_{\mathrm{B}}\right)$ ] will become $\approx \mathrm{n}\left(\mathrm{P}_{\mathrm{m}}+\mathrm{KP}_{\mathrm{B}}\right), C_{m}$ is compressed using Huffman Compression by considering the following two cases

(i) Both $(x, y)$ co-ordinates of the encrypted data of $\left[K G+n *\left(P_{m}+K P_{B}\right)\right]$ is compressed using Arithmetic/ Huffman compression and the results are shown in the corresponding tables and graphs [Table 4.1 to Table 4.4 and Figures 4.1 to Figures 4.2]. In this case, the amount of bandwidth saved is $50 \%$ of original encrypted data + reduced size of the compressed data. Hence,

$$
O B W S \%=\frac{0.5 * O E D S+C B \text { in }(x, y)}{O E D S} * 100
$$

*OEDS-Original encrypted data Size,

*CB-Compression Bits 
The overall percentage of the overall bandwidth required (OBWR) can be calculated by the equation

$$
\text { OBWR } \%=100-\text { OBWS } \%
$$

In this case, only $\mathrm{x}$ coordinates of encrypted data of $\left[K G+n *\left(P_{m}+K_{B}\right)\right]$ is taken for compression, as we know the $\mathrm{x}$-co-ordinate of the ECC, we can get the corresponding y co-ordinate by using the following cubic equation,

$$
\mathrm{y}^{2} \equiv \mathrm{x}^{3}+\mathrm{ax}+\mathrm{b} \bmod \mathrm{p}
$$

If we take only $x$ co-ordinate of the original encrypted data, then the amount of bandwidth saved is $75 \%$ of original encrypted data + reduced size of the compressed data. Hence, the percentage of the bandwidth saving (OBWS) can be calculated by the equation

$$
O B W S \%=\frac{0.75 * O E D S+C B \text { in }(x, y)}{O E D S} * 100
$$

The overall percentage of the bandwidth required (OBWR) can be calculated by the equation

\section{OBWR $\%=100-\mathrm{OBWS} \%$}

For this data, we computed the bandwidth required and saved by applying Arithmetic and Huffman compression. The results are shown in tables and graphs.

At the destination the data is uncompressed and original text is recovered by using the equation (2).

\section{ARITHMETIC COMPRESSION}

The following experiments are conducted by considering only $\mathrm{x}$ coordinates and both $(x, y)$ Coordinates of the encrypted text for computing compression ratio, overall percentage of bandwidth requirement, and saving for the following three cases.

\subsection{For $(x, y)$ Coordinates of the Encrypted Text}

This section presents the compression and compression ratio for three different cases

Table 1: Arithmetic Compression for Case (i)

\begin{tabular}{|c|l|c|c|c|c|c|}
\hline \multirow{2}{*}{$\begin{array}{c}\text { Sl. } \\
\text { No. }\end{array}$} & \multicolumn{3}{|c|}{ Input String } & \multirow{2}{*}{ OEDS } & \multirow{2}{*}{$(\mathbf{x , y})$} & \multicolumn{2}{|c|}{ Arithmetic } \\
\cline { 2 - 3 } \cline { 6 - 7 } & \multicolumn{1}{|c|}{ String } & $\begin{array}{c}\text { Size } \\
\text { (bits) }\end{array}$ & & EDS & CB & CR \\
\hline 1 & ABCD & 32 & 128 & 80 & 33 & 0.412 \\
\hline 2 & ABCDE & 40 & 160 & 96 & 42 & 0.437 \\
\hline 3 & ABCDEF & 48 & 192 & 112 & 53 & 0.473 \\
\hline 4 & ABCDEFG & 56 & 224 & 128 & 60 & 0.468 \\
\hline 5 & ABCDEFGH & 64 & 256 & 144 & 61 & 0.423 \\
\hline 6 & ABCDEFGHI & 72 & 288 & 160 & 61 & 0.381 \\
\hline 7 & ABCDEFGHIJ & 80 & 320 & 176 & 61 & 0.346 \\
\hline 8 & ABCDEFGHIJK & 88 & 352 & 192 & 62 & 0.322 \\
\hline 9 & ABCDEFGHIJKL & 96 & 384 & 208 & 61 & 0.293 \\
\hline 10 & ABCDEFGHIJKLM & 104 & 416 & 224 & 62 & 0.276 \\
\hline
\end{tabular}

\begin{tabular}{|c|c|c|c|c|c|c|}
\hline \multirow{2}{*}{$\begin{array}{l}\text { Sl. } \\
\text { No. }\end{array}$} & \multicolumn{2}{|c|}{ Input String } & \multirow[t]{2}{*}{ OEDS } & \multirow{2}{*}{$\begin{array}{c}(\mathbf{x}, \\
\mathbf{y}) \\
\text { EDS }\end{array}$} & \multicolumn{2}{|c|}{ Arithmetic } \\
\hline & String & Size & & & CB & CR \\
\hline 1 & APPLE & 40 & 160 & 96 & 39 & 0.406 \\
\hline 2 & COMPUTER & 64 & 256 & 144 & 61 & 0.423 \\
\hline 3 & DOCUMENT & 64 & 256 & 144 & 61 & 0.423 \\
\hline 4 & ELEPHANT & 64 & 256 & 144 & 59 & 0.409 \\
\hline 5 & GRAPHICS & 64 & 256 & 144 & 61 & 0.423 \\
\hline 6 & HARDDISK & 64 & 256 & 144 & 59 & 0.409 \\
\hline 7 & BEAUTIFUL & 72 & 288 & 160 & 61 & 0.381 \\
\hline 8 & FLOWCHART & 72 & 288 & 160 & 61 & 0.381 \\
\hline 9 & JNTUCEVZM & 72 & 288 & 160 & 61 & 0.381 \\
\hline 10 & INFORMATION & 88 & 352 & 192 & 59 & 0.307 \\
\hline
\end{tabular}

*EDS-Encrypted data size
Table 2: Arithmetic Compression for Case (ii)

Table 3: Arithmetic Compression for Case (iii)

\begin{tabular}{|c|l|c|c|c|c|c|}
\hline \multirow{2}{*}{$\begin{array}{c}\text { S.No } \\
\cdot\end{array}$} & \multicolumn{2}{|c|}{ Input String } & \multirow{2}{*}{ OED } & \multirow{2}{*}{$\mathbf{x , y})$} & \multicolumn{2}{|c|}{ Arithmetic } \\
\cline { 2 - 3 } & String & Size & S & EDS & C & CR \\
\hline 1 & AAAABCCDDDD & 88 & 352 & 192 & 56 & 0.29 \\
\hline 2 & ABCDEEEFFFG & 88 & 352 & 192 & 61 & 0.31 \\
\hline 3 & ABCDEEEEEEF & 88 & 352 & 192 & 61 & 0.31 \\
\hline 4 & DDEEFGHHHII & 88 & 352 & 192 & 59 & 0.30 \\
\hline 5 & KLKLFGHIJJ & 88 & 352 & 192 & 62 & 0.32 \\
\hline 6 & AABCCCDHIJK & 88 & 352 & 192 & 62 & 0.32 \\
\hline 7 & AAABBBCDEFJ & 88 & 352 & 192 & 59 & 0.30 \\
\hline 8 & AAAABBBBCCC & 88 & 352 & 192 & 60 & 0.31 \\
\hline 9 & AAAAAAAAAAAA & 96 & 384 & 208 & 36 & 0.17 \\
\hline 10 & AAAAAAABBBBB & 96 & 384 & 208 & 60 & 0.28 \\
\hline
\end{tabular}

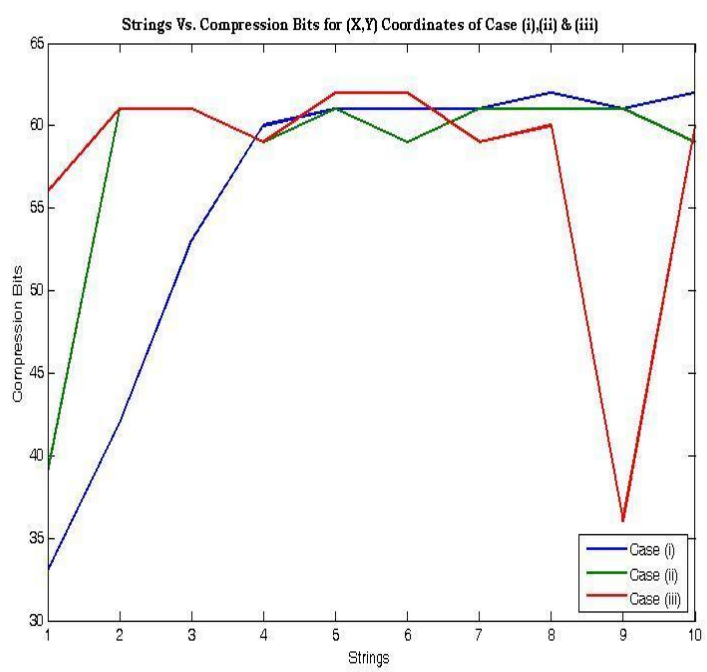

Figure 2: Compression Bits for Case (i),(ii)\& (ii) 


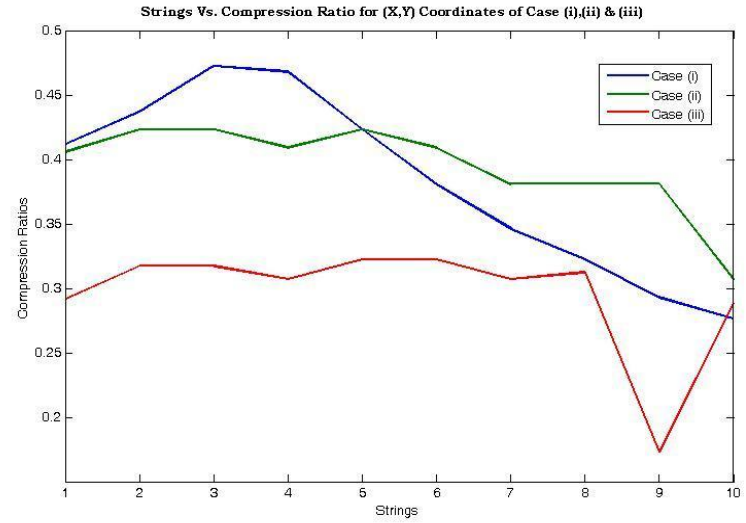

Figure 3: Compression Ratios for Case (i), (ii) \& (iii)

One can observe the following variation range in the compression bits and compression ratio from above Tables 1 to 3, Figures 2 and Figure 3

\begin{tabular}{|c|c|c|}
\hline Cases & Compression bits range & Compression Ratio range \\
\hline Case(i) & $33-62$ & $0.276-0.473$ \\
\hline Case(ii) & $39-61$ & $0.307-0.423$ \\
\hline Case(iii) & $36-62$ & $0.173-0.322$ \\
\hline
\end{tabular}

\subsection{Overall BWR\% and BWS\% in $(x, y)$ Coordinates:}

This section gives the overall percentage of bandwidth requirement and saved for above three cases by considering the both $(\mathrm{x}, \mathrm{y})$ co-ordinates of the encrypted data

Table 4 Overall BWR\% and BWS\% in Arithmetic Compression for case (i)

\begin{tabular}{|c|l|l|l|l|}
\hline SI. & \multicolumn{1}{|c|}{ Input String } & \multicolumn{1}{|c|}{ TBS } & OBWR\% & OBWS\% \\
\hline 1 & ABCD & 95 & 25.78125 & 74.21875 \\
\hline 2 & ABCDE & 118 & 26.25 & 73.75 \\
\hline 3 & ABCDEF & 139 & 27.60417 & 72.39583 \\
\hline 4 & ABCDEFG & 164 & 26.78571 & 73.21429 \\
\hline 5 & ABCDEFGH & 195 & 23.82813 & 76.17188 \\
\hline 6 & ABCDEFGHI & 227 & 21.18056 & 78.81944 \\
\hline 7 & ABCDEFGHIJ & 259 & 19.0625 & 80.9375 \\
\hline 8 & ABCDEFGHIJK & 290 & 17.61364 & 82.38636 \\
\hline 9 & ABCDEFGHIJKL & 323 & 15.88542 & 84.11458 \\
\hline 10 & ABCDEFGHIJKLM & 354 & 14.90385 & 85.09615 \\
\hline
\end{tabular}

$*$ TBS-total bits saved=(OEDS-CB $)$

Table 5 Overall BWR\% and BWS\% in Arithmetic Compression for case (ii)

\begin{tabular}{|c|l|c|c|c|}
\hline $\begin{array}{c}\text { Sl. } \\
\text { No. }\end{array}$ & Input String & TBS & OBWR\% & OBWS\% \\
\hline 1 & APPLE & 121 & 24.375 & 75.625 \\
\hline 2 & COMPUTER & 195 & 23.82813 & 76.171 \\
\hline 3 & DOCUMENT & 195 & 23.82813 & 76.171 \\
\hline 4 & ELEPHANT & 197 & 23.04688 & 76.953 \\
\hline 5 & GRAPHICS & 195 & 23.82813 & 76.171 \\
\hline
\end{tabular}

\begin{tabular}{|c|l|c|c|c|}
\hline 6 & HARDDISK & 197 & 23.04688 & 76.953 \\
\hline 7 & BEAUTIFUL & 227 & 21.18056 & 78.819 \\
\hline 8 & FLOWCHART & 227 & 21.18056 & 78.819 \\
\hline 9 & JNTUCEVZM & 227 & 21.18056 & 78.819 \\
\hline 10 & INFORMATION & 293 & 16.76136 & 83.23864 \\
\hline
\end{tabular}

Table 6 Overall BWR\% and BWS\% in Arithmetic Compression for case (iii)

\begin{tabular}{|c|l|c|c|c|}
\hline $\begin{array}{c}\text { Sl. } \\
\text { No. }\end{array}$ & \multicolumn{1}{|c|}{ Input String } & TBS & OBWR\% & OBWS\% \\
\hline 1 & AAAABCCDDDD & 296 & 15.90909 & 84.09091 \\
\hline 2 & ABCDEEEFFFG & 291 & 17.32955 & 82.67045 \\
\hline 3 & ABCDEEEEEEF & 291 & 17.32955 & 82.67045 \\
\hline 4 & DDEEFGHHHII & 293 & 16.76136 & 83.23864 \\
\hline 5 & KLKLFGHIJJ & 290 & 17.61364 & 82.38636 \\
\hline 6 & AABCCCDHIJK & 290 & 17.61364 & 82.38636 \\
\hline 7 & AAABBBCDEFJ & 293 & 16.76136 & 83.23864 \\
\hline 8 & AAAABBBBCCC & 292 & 17.04545 & 82.95455 \\
\hline 9 & AAAAAAAAAAAA & 348 & 9.375 & 90.625 \\
\hline 10 & AAAAAABBBBBB & 324 & 15.625 & 84.375 \\
\hline \multicolumn{5}{|r}{}
\end{tabular}

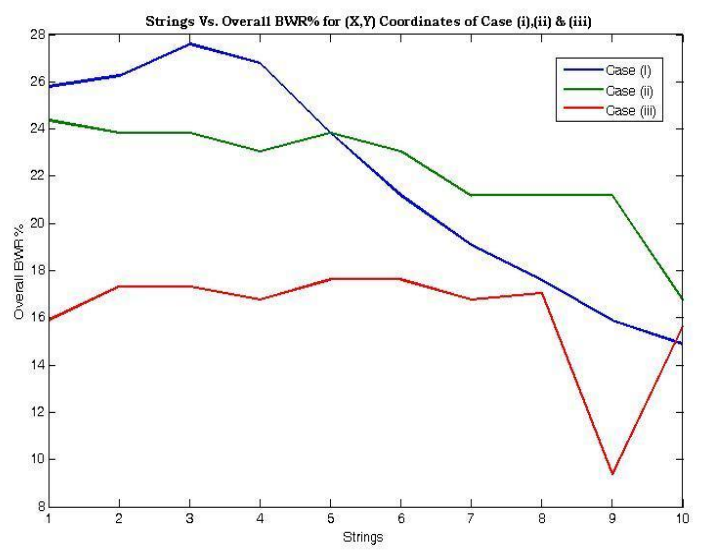

Figure 4: Overall BWR\% for Case (i),(ii)\& (iii)

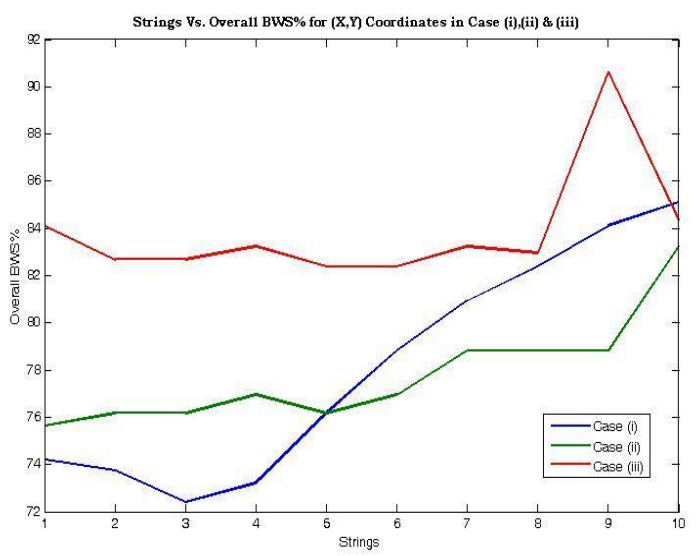

Figure 5 Overall BWS\% for Case (i), (ii) \& (iii) 
One can observe the following variation range in the overall BWR\% and BWS\% from above Tables 4 to 6 , Figures 4 and Figure 5

\begin{tabular}{|c|c|c|}
\hline Cases & OBWR\% Range & OBWS\% Range \\
\hline Case(i) & $14.90-27.60$ & $72.3-85.1$ \\
\hline Case(ii) & $16.76-24.375$ & $75.625-83.23$ \\
\hline Case(iii) & $9.375-17.61$ & $82.38-90.625$ \\
\hline
\end{tabular}

\subsection{For $(x)$ Coordinates of the encrypted data:}

This section gives the overall percentage of bandwidth requirement and saved for above three cases by considering only $x$ co-ordinates of the encrypted data.

Table 7: Arithmetic Compression for Case (i)

\begin{tabular}{|c|l|c|c|c|c|c|}
\hline \multirow{2}{*}{$\begin{array}{c}\text { Sl. } \\
\text { No. }\end{array}$} & \multicolumn{3}{|c|}{ Input String } & & & \multicolumn{2}{c|}{ Arithmetic } \\
\cline { 2 - 3 } \cline { 6 - 7 } & String & Size & OEDS & EDS & CB & CR \\
\hline 1 & ABCD & 32 & 128 & 40 & 12 & 0.3 \\
\hline 2 & ABCDE & 40 & 160 & 48 & 14 & 0.29166 \\
\hline 3 & ABCDEF & 48 & 192 & 56 & 19 & 0.33928 \\
\hline 4 & ABCDEFG & 56 & 224 & 64 & 22 & 0.34375 \\
\hline 5 & ABCDEFGH & 64 & 256 & 72 & 29 & 0.40277 \\
\hline 6 & ABCDEFGHI & 72 & 288 & 80 & 33 & 0.4125 \\
\hline 7 & ABCDEFGHIJ & 80 & 320 & 88 & 38 & 0.43181 \\
\hline 8 & ABCDEFGHIJK & 88 & 352 & 96 & 42 & 0.4375 \\
\hline 9 & ABCDEFGHIJKL & 96 & 384 & 104 & 46 & 0.44230 \\
\hline 10 & ABCDEFGHIJKLM & 104 & 416 & 112 & 53 & 0.47321 \\
\hline
\end{tabular}

Table 8: Arithmetic Compression for Case (ii)

\begin{tabular}{|c|l|c|c|c|c|c|}
\hline \multirow{2}{*}{$\begin{array}{c}\text { Sl. } \\
\text { No. }\end{array}$} & \multicolumn{2}{|c|}{ Input String } & & & \multicolumn{2}{c|}{ Arithmetic } \\
\cline { 2 - 3 } \cline { 6 - 7 } & String & Size & \multirow{2}{*}{ OEDS } & EDS & CB & CR \\
\hline 1 & APPLE & 40 & 160 & 48 & 14 & 0.291666 \\
\hline 2 & COMPUTER & 64 & 256 & 72 & 29 & 0.402777 \\
\hline 3 & DOCUMENT & 64 & 256 & 72 & 29 & 0.402777 \\
\hline 4 & ELEPHANT & 64 & 256 & 72 & 27 & 0.375 \\
\hline 5 & GRAPHICS & 64 & 256 & 72 & 29 & 0.402777 \\
\hline 6 & HARDDISK & 64 & 256 & 72 & 27 & 0.375 \\
\hline 7 & BEAUTIFUL & 72 & 288 & 80 & 32 & 0.4 \\
\hline 8 & FLOWCHART & 72 & 288 & 80 & 33 & 0.4125 \\
\hline 9 & JNTUCEVZM & 72 & 288 & 80 & 33 & 0.4125 \\
\hline 10 & INFORMATION & 88 & 352 & 96 & 37 & 0.385416 \\
\hline
\end{tabular}

Table 9: Arithmetic Compression for Case (iii)

\begin{tabular}{|c|c|c|c|c|c|c|}
\hline \multirow{2}{*}{$\begin{array}{l}\text { Sl. } \\
\text { No }\end{array}$} & \multicolumn{2}{|l|}{ Input String } & \multirow[b]{2}{*}{$\begin{array}{c}\text { OED } \\
S\end{array}$} & \multirow[b]{2}{*}{ EDS } & \multicolumn{2}{|c|}{ Arithmeti } \\
\hline & String & Size & & & $\begin{array}{l}\text { C } \\
\text { B }\end{array}$ & CR \\
\hline 1 & AAAABCCDDDD & 88 & 352 & 96 & 25 & 0.26 \\
\hline 2 & ABCDEEEFFFG & 88 & 352 & 96 & 33 & 0.34 \\
\hline 3 & ABCDEEEEEEF & 88 & 352 & 96 & 26 & 0.27 \\
\hline 4 & DDEEFGHHHII & 88 & 352 & 96 & 33 & 0.34 \\
\hline 5 & KLKLFGHIJJJ & 88 & 352 & 96 & 32 & 0.33 \\
\hline 6 & AABCCCDHIJK & 88 & 352 & 96 & 35 & 0.36 \\
\hline 7 & AAABBBCDEFJ & 88 & 352 & 96 & 32 & 0.33 \\
\hline
\end{tabular}

\begin{tabular}{|c|l|c|c|c|c|c|}
\hline 8 & AAAABBBBCCC & 88 & 352 & 96 & 20 & 0.03 \\
\hline 9 & AAAAAAAAAA & 96 & 384 & 104 & 4 & 0.16 \\
\hline 10 & AAAAAABBBBB & 96 & 384 & 104 & 17 & 0.22 \\
\hline
\end{tabular}

4.4 Overall BWR\% and BWS\% in $(x)$ Coordinates:

This section gives the overall percentage of bandwidth requirement and saved for above three cases by considering only $\mathrm{x}$ co-ordinates of the encrypted data

Table 10: Overall BWR\% and BWS\% in Arithmetic Compression case (i)

\begin{tabular}{|c|l|l|l|l|}
\hline $\begin{array}{c}\text { Sl. } \\
\text { No }\end{array}$ & Input String & $\begin{array}{c}\text { TBS(OED } \\
\text { S - CB) }\end{array}$ & $\begin{array}{c}\text { OBWR } \\
\mathbf{\%}\end{array}$ & $\begin{array}{c}\text { OBWS } \\
\mathbf{\%}\end{array}$ \\
\hline 1 & ABCD & 116 & 9.375 & 90.625 \\
\hline 2 & ABCDE & 146 & 8.75 & 91.25 \\
\hline 3 & ABCDEF & 173 & 9.895833 & 90.10417 \\
\hline 4 & ABCDEFG & 202 & 9.821429 & 90.17857 \\
\hline 5 & ABCDEFGH & 227 & 11.32813 & 88.67188 \\
\hline 6 & ABCDEFGHI & 255 & 11.45833 & 88.54167 \\
\hline 7 & ABCDEFGHIJ & 282 & 11.875 & 88.125 \\
\hline 8 & ABCDEFGHIJK & 310 & 11.93182 & 88.06818 \\
\hline 9 & ABCDEFGHIJKL & 338 & 11.97917 & 88.02083 \\
\hline 10 & ABCDEFGHIJKL & 363 & 12.74038 & 87.25962 \\
\hline
\end{tabular}

Table 11: Overall BWR\% and BWS\% in Arithmetic Compression case (ii)

\begin{tabular}{|c|l|c|c|c|}
\hline SI. No. & Input String & TBS & OBWR\% & OBWS\% \\
\hline 1 & APPLE & 146 & 8.75 & 91.25 \\
\hline 2 & COMPUTER & 227 & 11.32813 & 88.67188 \\
\hline 3 & DOCUMENT & 227 & 11.32813 & 88.67188 \\
\hline 4 & ELEPHANT & 229 & 10.54688 & 89.45313 \\
\hline 5 & GRAPHICS & 227 & 11.32813 & 88.67188 \\
\hline 6 & HARDDISK & 229 & 10.54688 & 89.45313 \\
\hline 7 & BEAUTIFUL & 256 & 11.11111 & 88.88889 \\
\hline 8 & FLOWCHART & 255 & 11.45833 & 88.54167 \\
\hline 9 & JNTUCEVZM & 255 & 11.45833 & 88.54167 \\
\hline 10 & INFORMATION & 251 & 12.84722 & 87.15278 \\
\hline
\end{tabular}

Table 12: Overall BWR\% and BWS\% in Arithmetic Compression case (iii)

\begin{tabular}{|c|l|c|c|c|}
\hline $\begin{array}{c}\text { Sl. } \\
\text { No. }\end{array}$ & Input String & TBS & OBWR\% & OBWS\% \\
\hline 1 & AAAABCCDDDD & 327 & 7.102273 & 92.89773 \\
\hline 2 & ABCDEEEFFFG & 319 & 9.375 & 90.625 \\
\hline 3 & ABCDEEEEEEF & 326 & 7.386364 & 92.61364 \\
\hline 4 & DDEEFGHHHII & 319 & 9.375 & 90.625 \\
\hline 5 & KLKLFGHIJJ & 320 & 9.090909 & 90.90909 \\
\hline 6 & AABCCCDHIJ & 317 & 9.943182 & 90.05682 \\
\hline 7 & AAABBBCDEFJ & 320 & 9.090909 & 90.90909 \\
\hline 8 & AAAABBBBCCC & 332 & 5.681818 & 94.31818 \\
\hline 9 & AAAAAAAAAAAA & 380 & 1.041667 & 98.95833 \\
\hline 10 & AAAAAABBBBBB & 367 & 4.427083 & 95.5729 \\
\hline
\end{tabular}




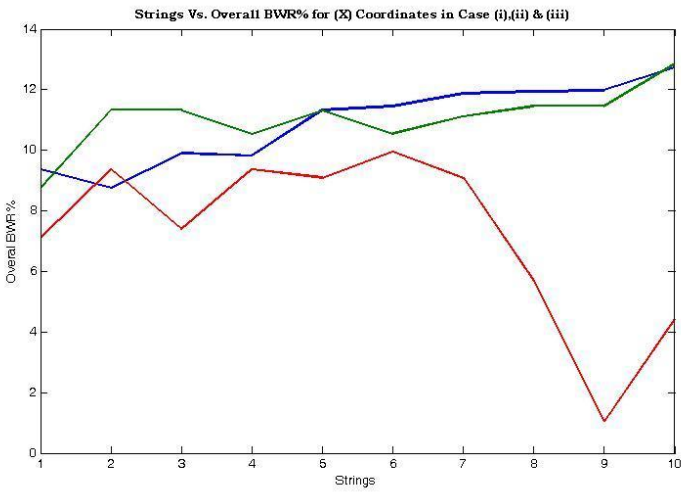

Figure 6: Overall BWR\% for Case (i),(ii) \& (iii)

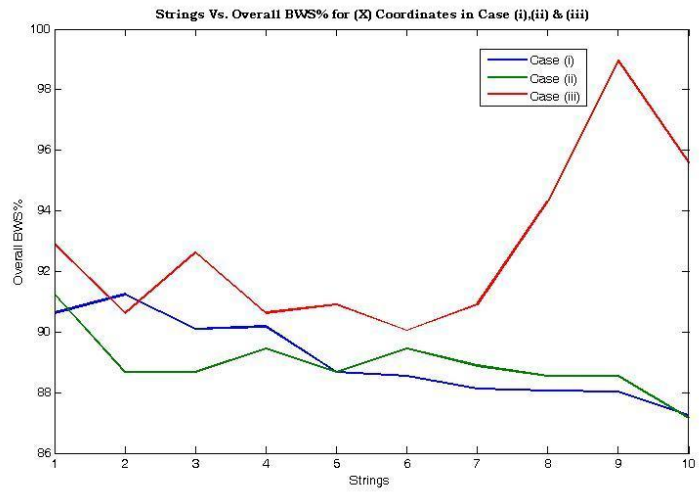

Figure 7 Overall BWS\% for Case (i), (ii) \& (iii)

One can observe the following variation range in the overall BWR\% and BWS\% from above Tables 10 to 12, Figures 6 and Figure 7 as follows.

\begin{tabular}{|c|c|c|}
\hline Cases & OBWR\% Range & OBWS\% Range \\
\hline Case(i) & $8.75-12.75$ & $87.25-91.25$ \\
\hline Case(ii) & $8.75-12.84$ & $87.15-91.25$ \\
\hline Case(iii) & $1.04-9.94$ & -98.95 \\
\hline
\end{tabular}

\section{HUFFMAN COMPRESSION:}

The following experiments are conducted by considering only $\mathrm{x}$ coordinates and both $(\mathrm{X}, \mathrm{Y})$ Coordinates of the encrypted text for computing compression ratio, the overall percentage of bandwidth required and saved for the following three cases.

\subsection{For $(x, y)$ Coordinates of the Encrypted Text:}

This section gives the overall percentage of bandwidth requirement and saved for above three cases by considering the both $(\mathrm{x}, \mathrm{y})$ co-ordinates of the encrypted data
Table 13: Huffman Compression for Case (i)

\begin{tabular}{|c|l|c|c|c|c|c|}
\hline \multirow{2}{*}{$\begin{array}{c}\text { SI. } \\
\text { No. }\end{array}$} & \multicolumn{2}{|c|}{ Input String } & & & \multicolumn{2}{c|}{ Huffman } \\
\hline 1 & String & Size & \multirow{2}{*}{ OEDS } & EDS & CB & CR \\
\hline 2 & ABCDE & 32 & 128 & 80 & 34 & 0.425 \\
\hline 3 & ABCDEF & 40 & 160 & 96 & 44 & 0.458 \\
\hline 4 & ABCDEFG & 48 & 192 & 112 & 54 & 0.482 \\
\hline 5 & ABCDEFGH & 56 & 224 & 128 & 64 & 0.5 \\
\hline 6 & ABCDEFGHI & 74 & 256 & 144 & 76 & 0.527 \\
\hline 7 & ABCDEFGHIJ & 80 & 320 & 176 & 100 & 0.568 \\
\hline 8 & ABCDEFGHIJK & 88 & 352 & 192 & 112 & 0.583 \\
\hline 9 & ABCDEFGHIJKL & 96 & 384 & 208 & 124 & 0.596 \\
\hline 10 & ABCDEFGHIJKLM & 104 & 416 & 224 & 136 & 0.607 \\
\hline
\end{tabular}

Table 14: Huffman Compression for Case (ii)

\begin{tabular}{|c|c|c|c|c|c|c|}
\hline \multirow{2}{*}{$\begin{array}{c}\text { Sl. } \\
\text { No } \\
\text {. }\end{array}$} & \multicolumn{2}{|c|}{ Input String } & \multirow[b]{2}{*}{$\begin{array}{c}\text { OED } \\
\text { S }\end{array}$} & \multirow{2}{*}{$\begin{array}{c}\text { EDS } \\
\text { (bits } \\
\text { ) }\end{array}$} & \multicolumn{2}{|c|}{ Huffman } \\
\hline & String & Size & & & CB & CR \\
\hline 1 & APPLE & 40 & 160 & 96 & 40 & 0.41 \\
\hline 2 & COMPUTER & 64 & 256 & 144 & 76 & 0.52 \\
\hline 3 & DOCUMENT & 64 & 256 & 144 & 74 & 0.51 \\
\hline 4 & ELEPHANT & 64 & 256 & 144 & 72 & 0.5 \\
\hline 5 & GRAPHICS & 64 & 256 & 144 & 76 & 0.52 \\
\hline 6 & HARDDISK & 64 & 256 & 144 & 72 & 0.5 \\
\hline 7 & BEAUTIFUL & 72 & 288 & 160 & 84 & 0.52 \\
\hline 8 & FLOWCHART & 72 & 288 & 160 & 90 & 0.56 \\
\hline 9 & JNTUCEVZM & 72 & 288 & 160 & 86 & 0.53 \\
\hline 10 & INFORMATIO & 88 & 352 & 192 & 100 & 0.52 \\
\hline
\end{tabular}

Table 15: Huffman Compression for Case (iii)

\begin{tabular}{|c|l|c|c|c|c|c|}
\hline & \multicolumn{2}{|c|}{ Input String } & & & \multicolumn{2}{c|}{ Huffman } \\
\cline { 2 - 3 } $\begin{array}{c}\text { Sl. } \\
\text { No. }\end{array}$ & \multicolumn{1}{|c|}{ String } & Size & & \multirow{2}{*}{$\begin{array}{c}\text { EDS } \\
\text { (bits) }\end{array}$} & CB & CR \\
\hline 1 & AAAABCCDDDD & 88 & 352 & 192 & 76 & 0.395 \\
\hline 2 & ABCDEEEFFFG & 88 & 352 & 192 & 92 & 0.479 \\
\hline 3 & ABCDEEEEEEF & 88 & 352 & 192 & 80 & 0.416 \\
\hline 4 & DDEEFGHHHII & 88 & 352 & 192 & 90 & 0.467 \\
\hline 5 & KLKLFGHIJJ & 88 & 352 & 192 & 94 & 0.489 \\
\hline 6 & AABCCCDHIJ & 88 & 352 & 192 & 98 & 0.510 \\
\hline 7 & AAABBBCDEFJ & 88 & 352 & 192 & 92 & 0.479 \\
\hline 8 & AAAABBBBCCC & 88 & 352 & 192 & 70 & 0.364 \\
\hline 9 & AAAAAAAAAAAAA & 96 & 384 & 208 & 42 & 0.201 \\
\hline 10 & AAAAAAABBBBBB & 96 & 384 & 208 & 62 & 0.298 \\
\hline
\end{tabular}




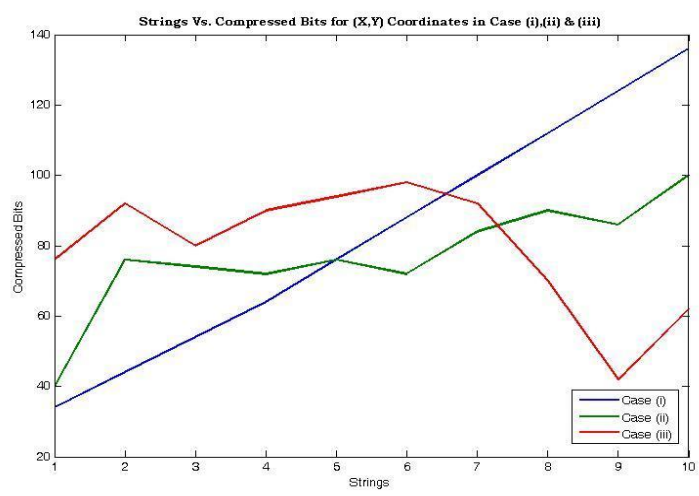

Figure 8: Compressed Bits for Case (i),(ii) \& (iii)

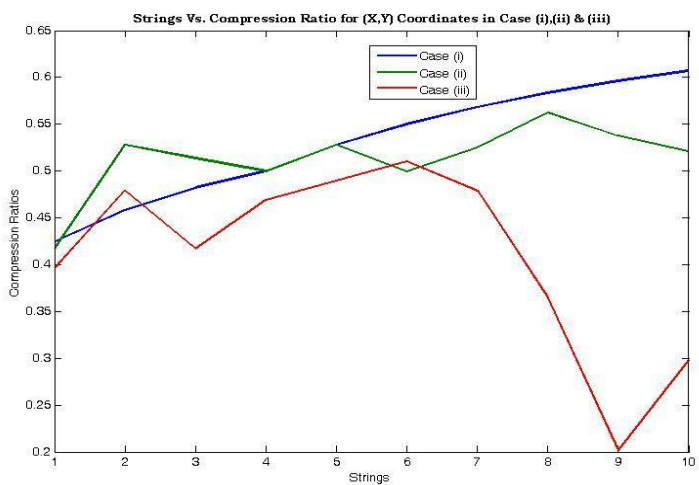

Figure 9: Compressed Ratio for Case (i), (ii) \& (iii)

One can observe the following variation range in the compression bits and compression ratio from above the Tables 13 to 15 , Figures 8 and Figure 9

\begin{tabular}{|c|c|c|}
\hline Cases & Compression bits & Compression Ratio \\
\hline Case(i) & $34-136$ & $0.425-0.607$ \\
\hline Case(ii) & $40-100$ & $0.41-0.56$ \\
\hline Case(iii) & $42-98$ & $0.201-0.51$ \\
\hline
\end{tabular}

\subsection{Overall BWR\% and BWS\% in $(x, y)$ Coordinates:}

This section gives the overall percentage of bandwidth requirement and saved for above three cases by considering the both $(\mathrm{x}, \mathrm{y})$ co-ordinates of the encrypted data

Table 16 Overall BWR\% and BWS\% in Huffman Compression Case (i)

\begin{tabular}{|c|l|l|l|l|}
\hline $\begin{array}{c}\text { Sl. } \\
\text { No }\end{array}$ & \multicolumn{1}{|c|}{ Input String } & $\begin{array}{c}\text { TBS(OED } \\
\text { S - CB) }\end{array}$ & $\begin{array}{c}\text { OBWR } \\
\text { \% }\end{array}$ & $\begin{array}{c}\text { OBWS } \\
\text { \% }\end{array}$ \\
\hline 1 & ABCD & 94 & 26.5625 & 73.4375 \\
\hline 2 & ABCDE & 116 & 27.5 & 72.5 \\
\hline 3 & ABCDEF & 138 & 28.125 & 71.875 \\
\hline 4 & ABCDEFG & 160 & 28.57143 & 71.42857 \\
\hline 5 & ABCDEFGH & 180 & 29.6875 & 70.3125 \\
\hline 6 & ABCDEFGHI & 200 & 30.55556 & 69.44444 \\
\hline
\end{tabular}

\begin{tabular}{|c|l|l|l|l|}
\hline 7 & ABCDEFGHIJ & 220 & 31.25 & 68.75 \\
\hline 8 & ABCDEFGHIJ & 240 & 31.81818 & 68.18182 \\
\hline 9 & ABCDEFGHIJKL & 260 & 32.29167 & 67.70833 \\
\hline 10 & ABCDEFGHIJKL & 280 & 32.69231 & 67.30769 \\
\hline
\end{tabular}

Table 17 Overall BWR\% and BWS\% in Huffman Compression Case (ii)

\begin{tabular}{|c|l|c|c|c|}
\hline $\begin{array}{c}\text { Sl. } \\
\text { No. }\end{array}$ & Input String & $\begin{array}{c}\text { TBS(OEDS } \\
-\mathbf{C B})\end{array}$ & OBWR\% & OBWS\% \\
\hline 1 & APPLE & 120 & 25 & 75 \\
\hline 2 & COMPUTER & 180 & 29.6875 & 70.3125 \\
\hline 3 & DOCUMENT & 182 & 28.90625 & 71.09375 \\
\hline 4 & ELEPHANT & 184 & 28.125 & 71.875 \\
\hline 5 & GRAPHICS & 180 & 29.6875 & 70.3125 \\
\hline 6 & HARDDISK & 184 & 28.125 & 71.875 \\
\hline 7 & BEAUTIFUL & 204 & 29.16667 & 70.83333 \\
\hline 8 & FLOWCHART & 198 & 31.25 & 68.75 \\
\hline 9 & JNTUCEVZM & 202 & 29.86111 & 70.13889 \\
\hline 10 & INFORMATION & 252 & 28.40909 & 71.59091 \\
\hline
\end{tabular}

Table 18: Overall BWR\% and BWS\% in Huffman Compression Case (iii)

\begin{tabular}{|c|l|c|c|c|}
\hline $\begin{array}{c}\text { Sl. } \\
\text { No }\end{array}$ & \multicolumn{1}{|c|}{ Input String } & $\begin{array}{c}\text { TBS(OED } \\
\text { S }-\mathbf{C B})\end{array}$ & $\begin{array}{c}\text { OBWR } \\
\mathbf{\%}\end{array}$ & $\begin{array}{c}\text { OBWS } \\
\mathbf{\%}\end{array}$ \\
\hline 1 & AAAABCCDDDD & 276 & 21.59091 & 78.409 \\
\hline 2 & ABCDEEEFFFG & 260 & 26.13636 & 73.863 \\
\hline 3 & ABCDEEEEEEF & 272 & 22.72727 & 77.27 \\
\hline 4 & DDEEFGHHHII & 262 & 25.56818 & 74.43 \\
\hline 5 & KLKLFGHIJJ & 258 & 26.70455 & 73.295 \\
\hline 6 & AABCCCDHIJK & 254 & 27.84091 & 72.159 \\
\hline 7 & AAABBBCDEFJ & 260 & 26.13636 & 73.863 \\
\hline 8 & AAAABBBBCCC & 282 & 19.88636 & 80.113 \\
\hline 9 & AAAAAAAAAAA & 342 & 10.9375 & 89.062 \\
\hline 10 & AAAAAABBBBB & 322 & 16.14583 & 83.854 \\
\hline
\end{tabular}

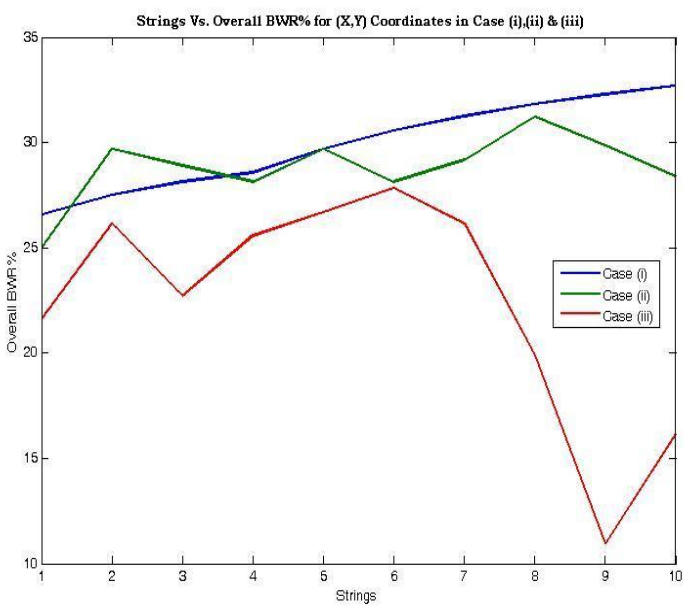

Figure 10: Overall BWR\% for Case (i), (ii) \& (iii) 


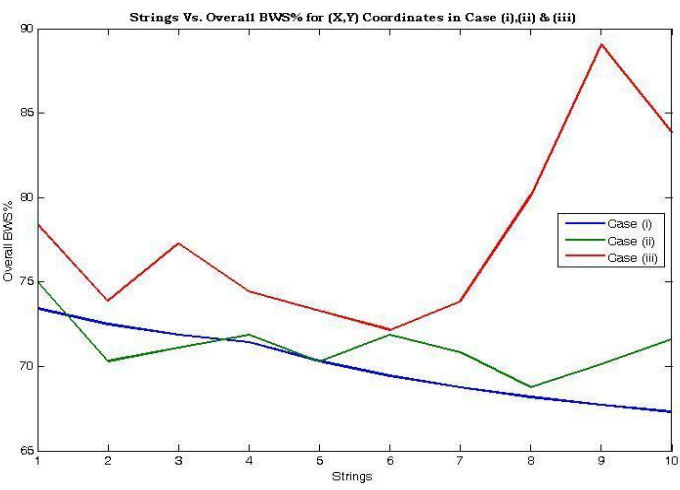

Figure 11: Overall BWS\% for Case (i), (ii) \& (iii)

One can observe the following variation range the overall BWR\% and BWS\% from the above Tables 5.4 to 5.6, Figures 5.3 and Figure 5.4 .

\begin{tabular}{|c|c|c|}
\hline Cases & Overall BWR\% & Overall BWS\% \\
\hline Case(i) & $26.56-32.69$ & $67.30-73.43$ \\
\hline Case(ii) & $25-31.25$ & $68.75-75$ \\
\hline Case(iii) & $10.93-27.84$ & $72.15-89.06$ \\
\hline
\end{tabular}

\subsection{For $(X)$ Coordinates encrypted text data:}

This section gives the overall percentage of bandwidth requirement and saved for above three cases by considering only $\mathrm{X}$ co-ordinates of the encrypted data

Table 19: Huffman Compression for Case (i)

\begin{tabular}{|c|c|c|c|c|c|c|}
\hline \multirow[b]{2}{*}{$\begin{array}{l}\text { Sl. } \\
\text { No. }\end{array}$} & \multicolumn{2}{|l|}{ Input String } & \multirow[b]{2}{*}{ OEDS } & \multirow[b]{2}{*}{$\begin{array}{c}\text { EDS } \\
\text { (bits) }\end{array}$} & \multicolumn{2}{|c|}{ Huffman } \\
\hline & String & Size & & & CB & CR \\
\hline 1 & $\mathrm{ABCD}$ & 32 & 128 & 40 & 12 & 0.3 \\
\hline 2 & $\mathrm{ABCDE}$ & 40 & 160 & 48 & 16 & 0.333 \\
\hline 3 & ABCDEF & 48 & 192 & 56 & 20 & 0.357 \\
\hline 4 & ABCDEFG & 56 & 224 & 64 & 24 & 0.375 \\
\hline 5 & ABCDEFGH & 64 & 256 & 72 & 29 & 0.402 \\
\hline 6 & ABCDEFGHI & 72 & 288 & 80 & 34 & 0.425 \\
\hline 7 & ABCDEFGHIJ & 80 & 320 & 88 & 39 & 0.443 \\
\hline 8 & ABCDEFGHIJK & 88 & 352 & 96 & 44 & 0.458 \\
\hline 9 & ABCDEFGHIJKL & 96 & 384 & 104 & 49 & 0.471 \\
\hline 10 & ABCDEFGHIJKLM & 104 & 416 & 112 & 54 & 0.482 \\
\hline
\end{tabular}

Table 20: Huffman Compression for Case (ii)

\begin{tabular}{|c|l|c|c|l|l|l|}
\hline & \multicolumn{2}{|c|}{ Input String } & & & \multicolumn{2}{c|}{ Huffman } \\
\cline { 7 - 8 } \cline { 6 - 7 } $\begin{array}{c}\text { Sl. } \\
\text { No. }\end{array}$ & String & Size & & EDS & & \\
\hline 1 & APPLE & 40 & 160 & 48 & 14 & 0.291666 \\
\hline 2 & COMPUTER & 64 & 256 & 72 & 29 & 0.402777 \\
\hline 3 & DOCUMENT & 64 & 256 & 72 & 29 & 0.402777 \\
\hline 4 & ELEPHANT & 64 & 256 & 72 & 27 & 0.375 \\
\hline 5 & GRAPHICS & 64 & 256 & 72 & 29 & 0.402777 \\
\hline 6 & HARDDISK & 64 & 256 & 72 & 27 & 0.375 \\
\hline 7 & BEAUTIFUL & 72 & 288 & 80 & 32 & 0.4 \\
\hline
\end{tabular}

\begin{tabular}{|c|l|c|c|c|c|l|}
\hline 8 & FLOWCHART & 72 & 288 & 80 & 34 & 0.425 \\
\hline 9 & JNTUCEVZM & 72 & 288 & 80 & 34 & 0.425 \\
\hline 10 & INFORMATION & 88 & 352 & 96 & 38 & 0.39583 \\
\hline
\end{tabular}

Table 21: Huffman Compression for Case (iii)

\begin{tabular}{|c|c|c|c|c|c|c|}
\hline \multirow{2}{*}{$\begin{array}{l}\text { Sl. } \\
\text { No }\end{array}$} & \multicolumn{2}{|l|}{ Input String } & \multirow[b]{2}{*}{$\begin{array}{c}\text { OED } \\
\text { S }\end{array}$} & \multirow{2}{*}{$\begin{array}{c}\text { EDS } \\
\text { (bits } \\
\text { ) }\end{array}$} & \multicolumn{2}{|c|}{ Huffman } \\
\hline & String & Size & & & $\begin{array}{l}\text { C } \\
\text { B }\end{array}$ & CR \\
\hline 1 & AAAABCCDDDD & 88 & 352 & 96 & 26 & 0.27 \\
\hline 2 & ABCDEEEFFFG & 88 & 352 & 96 & 34 & 0.35 \\
\hline 3 & ABCDEEEEEEF & 88 & 352 & 96 & 28 & 0.29 \\
\hline 4 & DDEEFGHHHII & 88 & 352 & 96 & 33 & 0.34 \\
\hline 5 & KLKLFGHIJJ & 88 & 352 & 96 & 35 & 0.36 \\
\hline 6 & AABCCCDHIJK & 88 & 352 & 96 & 37 & 0.38 \\
\hline 7 & AAABBBCDEFJ & 88 & 352 & 96 & 34 & 0.35 \\
\hline 8 & AAAABBBBCCC & 88 & 352 & 96 & 24 & 0.12 \\
\hline 9 & AAAAAAAAAAA & 96 & 384 & 104 & 13 & 0.19 \\
\hline 10 & AAAAAABBBBB & 96 & 384 & 104 & 20 & 0.25 \\
\hline
\end{tabular}

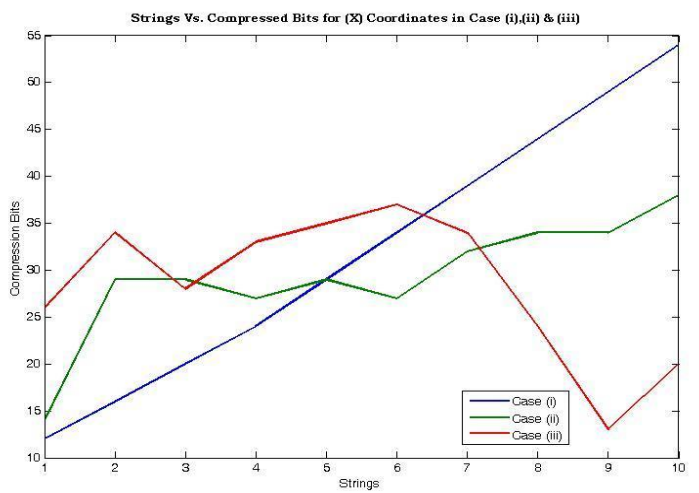

Figure 12: Compressed Bits for Case (i), (ii) \& (iii)

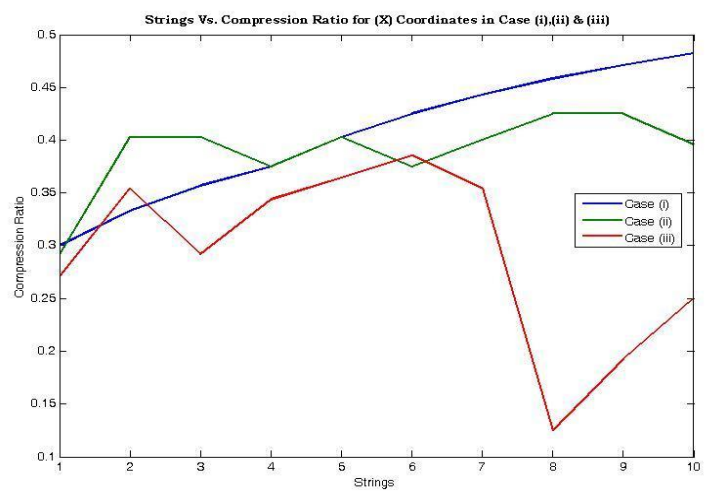

Figure 13: Compressed Ratio for Case (i), (ii) \& (iii) 
One can observe the following variation range in the compression bits and compression ratio from the above Tables 19 to 21 , Figures 12 and Figure 13

\begin{tabular}{|c|c|c|}
\hline Cases & Compression bits & Compression Ratio \\
\hline Case(i) & $12-54$ & $0.3-0.48$ \\
\hline Case(ii) & $14-38$ & $0.29-0.425$ \\
\hline Case(iii) & $13-37$ & $0.125-0.385$ \\
\hline
\end{tabular}

\subsection{Overall BWR\% and BWS\% in $x$ Coordinates:}

This section gives the overall percentage of bandwidth requirement and saved for above three cases by considering only $\mathrm{x}$ co-ordinates of the encrypted data

Table 22: Overall BWR\% and BWS \% in Huffman Compression Case (i)

\begin{tabular}{|c|l|l|l|l|}
\hline $\begin{array}{c}\text { Sl. } \\
\text { No. }\end{array}$ & \multicolumn{1}{|c|}{ Input String } & TBS & OBWR\% & OBWS\% \\
\hline 1 & ABCD & 116 & 9.375 & 90.625 \\
\hline 2 & ABCDE & 144 & 10 & 90 \\
\hline 3 & ABCDEF & 172 & 10.41667 & 89.583 \\
\hline 4 & ABCDEFG & 200 & 10.71429 & 89.285 \\
\hline 5 & ABCDEFGH & 227 & 11.32813 & 88.671 \\
\hline 6 & ABCDEFGHI & 254 & 11.80556 & 88.194 \\
\hline 7 & ABCDEFGHIJ & 281 & 12.1875 & 87.812 \\
\hline 8 & ABCDEFGHIJK & 308 & 12.5 & 87.5 \\
\hline 9 & ABCDEFGHIJKL & 335 & 12.76042 & 87.239 \\
\hline 10 & ABCDEFGHIJKLM & 362 & 13.94231 & 86.057 \\
\hline
\end{tabular}

Table 23: Overall BWR\% and BWS\% in Huffman Compression Case (ii)

\begin{tabular}{|c|l|l|l|l|}
\hline $\begin{array}{c}\text { Sl. } \\
\text { No. }\end{array}$ & Input String & TBS & OBWR\% & OBWS\% \\
\hline 1 & APPLE & 146 & 8.75 & 91.25 \\
\hline 2 & COMPUTER & 227 & 11.32813 & 88.67188 \\
\hline 3 & DOCUMENT & 227 & 11.32813 & 88.67188 \\
\hline 4 & ELEPHANT & 229 & 10.54688 & 89.45313 \\
\hline 5 & GRAPHICS & 227 & 11.32813 & 88.67188 \\
\hline 6 & HARDDISK & 229 & 10.54688 & 89.45313 \\
\hline 7 & BEAUTIFUL & 256 & 11.11111 & 88.88889 \\
\hline 8 & FLOWCHART & 254 & 11.80556 & 88.19444 \\
\hline 9 & JNTUCEVZM & 254 & 15.97222 & 84.02778 \\
\hline 10 & INFORMATION & 314 & 10.7954 & 89.2045 \\
\hline
\end{tabular}

Table 24: Overall BWR\% and BWS\% in Huffman Compression Case (iii)

\begin{tabular}{|c|l|c|c|c|}
\hline $\begin{array}{c}\text { Sl. } \\
\text { No }\end{array}$ & \multicolumn{1}{|c|}{ Input String } & $\begin{array}{c}\text { TB } \\
\text { S }\end{array}$ & $\begin{array}{c}\text { OBWR } \\
\mathbf{\%}\end{array}$ & $\begin{array}{c}\text { OBWS } \\
\mathbf{\%}\end{array}$ \\
\hline 1 & AAAABCCDDDD & 326 & 7.386364 & 92.61364 \\
\hline 2 & ABCDEEEFFFG & 318 & 9.659091 & 90.34091 \\
\hline 3 & ABCDEEEEEEF & 324 & 7.954545 & 92.04545 \\
\hline 4 & DDEEFGHHHII & 319 & 9.375 & 90.625 \\
\hline
\end{tabular}

\begin{tabular}{|c|l|c|c|c|}
\hline 5 & KLKLFGHIJJ & 317 & 9.943182 & 90.05682 \\
\hline 6 & AABCCCDHIJ & 315 & 10.51136 & 89.48864 \\
\hline 7 & AAABBBCDEFJ & 318 & 9.659091 & 90.34091 \\
\hline 8 & AAAABBBBCCC & 328 & 6.818182 & 93.18182 \\
\hline 9 & AAAAAAAAAAA & 371 & 3.385417 & 96.61458 \\
\hline 10 & AAAAAABBBBB & 364 & 5.208333 & 94.79167 \\
\hline
\end{tabular}

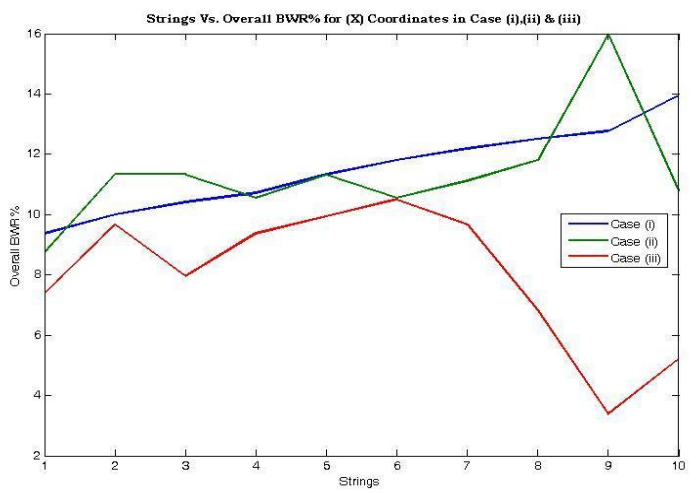

Figure 14 : Overall BWR\% for Case (i), (ii) \& (iii)

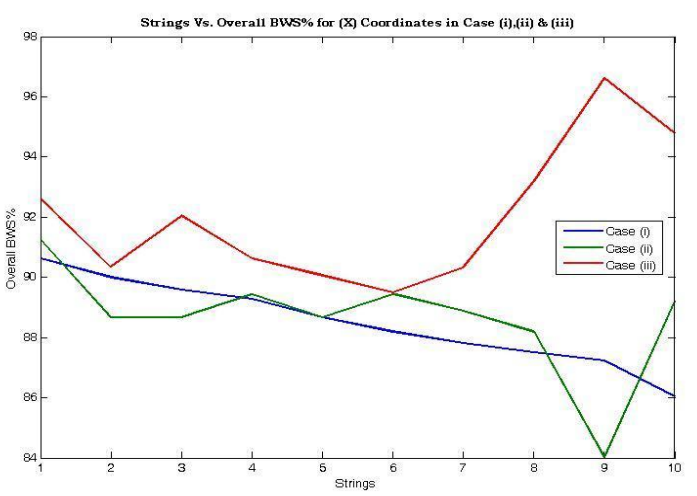

Figure 15: Overall BWS\% for Case (i), (ii) \& (iii)

One can observe the following variation range in the overall BWR $\%$ and BWS \% from the above Tables 22 to 24 and Figures 14 and Figure 15 as follows.

\begin{tabular}{|c|c|c|}
\hline Cases & OBWR\% Range & OBWS\% Range \\
\hline Case(i) & $9.375-13.94$ & $86.05-90.625$ \\
\hline Case(ii) & $8.75-15.97$ & $84.02-91.25$ \\
\hline Case(iii) & $3.38-10.51$ & $89.48-96.61$ \\
\hline
\end{tabular}




\section{ANALYSIS OF ARITHMETIC AND HUFFMAN COMPRESSION FOR OVERALL BWR\% AND BWS\%}

This section compares the performance of Arithmetic and Huffman compression Techniques

\subsection{For $(x, y)$ Coordinates of the encrypted data:}

This section gives the overall percentage of bandwidth requirement and saved for above three cases by considering the both $(\mathrm{x}, \mathrm{y})$ co-ordinates of the encrypted data

Table 25: Overall BWR\% and BWS\% for case $(i)$

\begin{tabular}{|c|l|l|l|l|l|}
\hline S & Input & \multicolumn{2}{|c|}{ BWR\% } & \multicolumn{2}{c|}{ BWS\% } \\
\cline { 3 - 6 } $\mathbf{l .}$ & String & Arith & Huff & Arith & Huff \\
\hline 1 & ABCD & 25.78 & 26.5 & 74.21 & 73.4 \\
\hline 2 & ABCDE & 26.25 & 27.5 & 73.75 & 72.5 \\
\hline 3 & ABCDEF & 27.60 & 28.1 & 72.39 & 71.8 \\
\hline 4 & ABCDEF & 26.78 & 28.5 & 73.21 & 71.4 \\
\hline 5 & ABCDEF & 23.82 & 29.6 & 76.17 & 70.3 \\
\hline 6 & ABCDEF & 21.18 & 30.5 & 78.81 & 69.4 \\
\hline 7 & ABCDEF & 19.06 & 31.2 & 80.93 & 68.7 \\
\hline 8 & ABCDEF & 17.61 & 31.8 & 82.38 & 68.1 \\
\hline 9 & ABCDEF & 15.88 & 32.2 & 84.11 & 67.7 \\
\hline 1 & ABCDEF & 14.90 & 32.6 & 85.09 & 67.3 \\
\hline
\end{tabular}

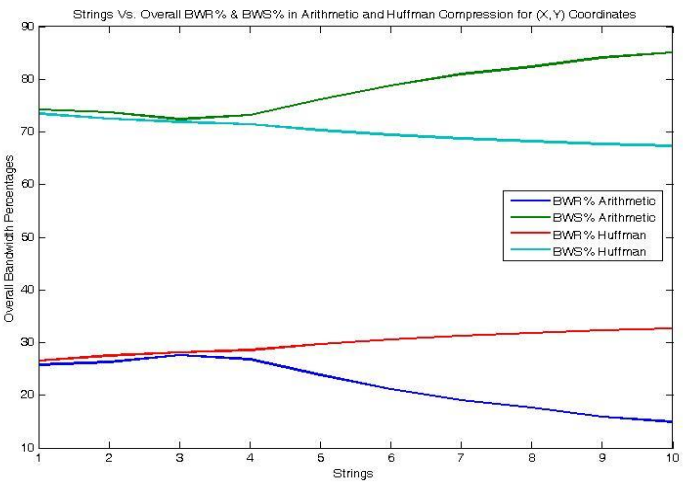

Figure 16: Overall BWR\% \& BWS\%

From the above Table 25and Figure 16, one can observe that, the overall percentage variation range in the bandwidth requirement and saving as follows:

\begin{tabular}{|l|l|l|l|}
\hline S.No. & Compression Type & \multicolumn{1}{|c|}{ BWR\% range } & BWS\% range \\
\hline 1 & Arithmetic & $14.92-27.6$ & $72.39-85.09$ \\
\hline 2 & Huffman & $26.56-32.69$ & $67.32-73.43$ \\
\hline
\end{tabular}

Table 26: Overall BWR\% and BWS\% for case (ii)

\begin{tabular}{|c|l|c|c|c|c|}
\hline \multirow{2}{*}{$\begin{array}{c}\text { Sl. } \\
\text { No. }\end{array}$} & \multirow{2}{*}{ Input String } & \multicolumn{2}{|c|}{ OBWR\% } & \multicolumn{2}{c|}{ OBWS\% } \\
\cline { 3 - 6 } & & Arithmetic & Huffman & Arithmetic & Huffman \\
\hline 1 & APPLE & 24.375 & 25 & 75.625 & 75 \\
\hline 2 & COMPUTER & 23.82813 & 29.6875 & 76.1718 & 70.312 \\
\hline 3 & DOCUMENT & 23.82813 & 28.90625 & 76.1718 & 71.093 \\
\hline 4 & ELEPHANT & 23.04688 & 28.125 & 76.9531 & 71.87 \\
\hline 5 & GRAPHICS & 23.82813 & 29.6875 & 76.1718 & 70.312 \\
\hline 6 & HARDDISK & 23.04688 & 28.125 & 76.9531 & 71.875 \\
\hline 7 & BEAUTIFUL & 21.18056 & 29.16667 & 78.8194 & 70.833 \\
\hline 8 & FLOWCHART & 21.18056 & 31.25 & 78.8194 & 68.75 \\
\hline 9 & JNTUCEVZM & 21.18056 & 29.86111 & 78.8194 & 70.138 \\
\hline 10 & INFORMATION & 16.76136 & 28.40909 & 83.2386 & 71.590 \\
\hline
\end{tabular}

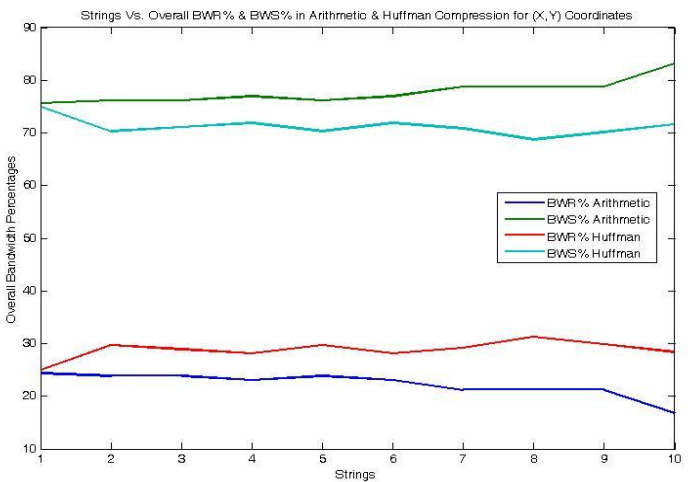

Figure 17: Overall BWR\% \& BWS\%

From the above Table 26 and Figure 17, one can observe that, the overall percentage variation range in the bandwidth requirement and saving as follows:

\begin{tabular}{|l|l|c|c|}
\hline S.No. & Compression Type & BWR\% range & BWS\% range \\
\hline 1 & Arithmetic & $16.76-24.375$ & $75.625-83.23$ \\
\hline 2 & Huffman & $25-31.25$ & $68.75-75$ \\
\hline
\end{tabular}

Table 27: Overall BWR\% and BWS\% for case (iii)

\begin{tabular}{|c|l|c|c|c|c|}
\hline \multirow{2}{*}{$\begin{array}{c}\text { Sl. } \\
\mathbf{N} \\
\mathbf{o}\end{array}$} & \multirow{2}{*}{ Input String } & $\mathbf{O B W R} \%$ & $\begin{array}{c}\text { OBWS } \\
\mathbf{\%}\end{array}$ & $\mathbf{O B W R} \%$ & $\begin{array}{c}\text { OBWS } \\
\mathbf{\%}\end{array}$ \\
\cline { 3 - 6 } & & $\begin{array}{c}\text { Arithmeti } \\
\mathbf{c}\end{array}$ & $\mathbf{H u f f m a n}$ & $\begin{array}{c}\text { Arithmeti } \\
\mathbf{c}\end{array}$ & Huffman \\
\hline 1 & $\begin{array}{c}\text { AAAAAAAAAAA } \\
\text { A }\end{array}$ & 15.90909 & 21.5909 & 84.09091 & 78.4090 \\
\hline 2 & AAAAAABBBBBB & 17.32955 & 26.1363 & 82.67045 & 73.8636 \\
\hline 3 & AAAABBBBCCCC & 17.32955 & 22.7272 & 82.67045 & 77.2727 \\
\hline 4 & AAABBBCCCDDD & 16.76136 & 25.5681 & 83.23864 & 74.4318 \\
\hline 5 & AABBCCDDEEFF & 17.61364 & 26.7045 & 82.38636 & 73.2954 \\
\hline 6 & AABBCCDDDEEE & 17.61364 & 27.8409 & 82.38636 & 72.1590 \\
\hline 7 & AAAABCCDDDD & 16.76136 & 26.1363 & 83.23864 & 73.8636 \\
\hline 8 & ABCDEEEFFFG & 17.04545 & 19.8863 & 82.95455 & 80.1136 \\
\hline 9 & ABCDEEEEEEF & 9.375 & 10.9375 & 90.625 & 89.0625 \\
\hline 10 & DDEEFGHHHII & 15.625 & 16.1458 & 84.375 & 83.8541 \\
\hline
\end{tabular}




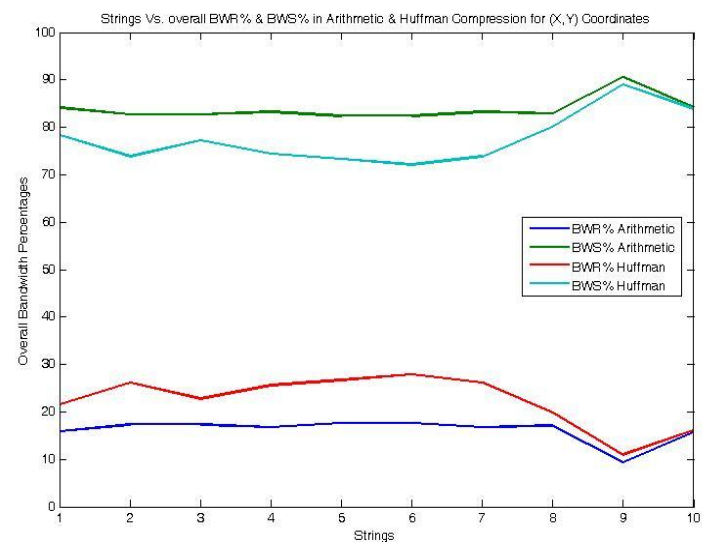

Figure 18: Overall BWR\% \& BWS\%

From the above Table 27 and Figure 18, one can observe that, the overall percentage variation range in the bandwidth requirement and saving as follows:

\begin{tabular}{|l|l|l|l|}
\hline S.No. & Compression Type & \multicolumn{1}{c|}{ BWR\% range } & BWS\% range \\
\hline & Arithmetic & $9.375-17.61$ & $82.38-90.625$ \\
\hline 2 & Huffman & $10.93-27.84$ & $72.15-89.06$ \\
\hline
\end{tabular}

\subsection{For $(x)$ Coordinates of the encrypted data:}

This section gives the overall percentage of bandwidth requirement and saved for above three cases by considering only $\mathrm{X}$ co-ordinates of the encrypted data

Table 28: Overall BWR\% and BWS\% for case(i)

\begin{tabular}{|c|l|c|c|c|c|}
\hline \multirow{2}{*}{$\begin{array}{c}\text { Sl. } \\
\text { No. }\end{array}$} & \multirow{2}{*}{ Input String } & \multicolumn{2}{|c|}{ BWR\% } & \multicolumn{2}{c|}{ BWS\% } \\
\cline { 3 - 6 } & & Arithmetic & Huffman & Arithmetic & Huffman \\
\hline 1 & ABCD & 9.375 & 9.375 & 90.625 & 90.625 \\
\hline 2 & ABCDE & 8.75 & 10 & 91.25 & 90 \\
\hline 3 & ABCDEF & 9.895833 & 10.41667 & 90.10417 & 89.58333 \\
\hline 4 & ABCDEFG & 9.821429 & 10.71429 & 90.17857 & 89.28571 \\
\hline 5 & ABCDEFGH & 11.32813 & 11.32813 & 88.67188 & 88.67188 \\
\hline 6 & ABCDEFGHI & 11.45833 & 11.80556 & 88.54167 & 88.19444 \\
\hline 7 & ABCDEFGHIJ & 11.875 & 12.1875 & 88.125 & 87.8125 \\
\hline 8 & ABCDEFGHIJK & 11.93182 & 12.5 & 88.06818 & 87.5 \\
\hline 9 & ABCDEFGHIJKL & 11.97917 & 12.76042 & 88.02083 & 87.23958 \\
\hline 10 & ABCDEFGHIJKLM & 12.74038 & 13.94231 & 87.25962 & 86.05769 \\
\hline
\end{tabular}

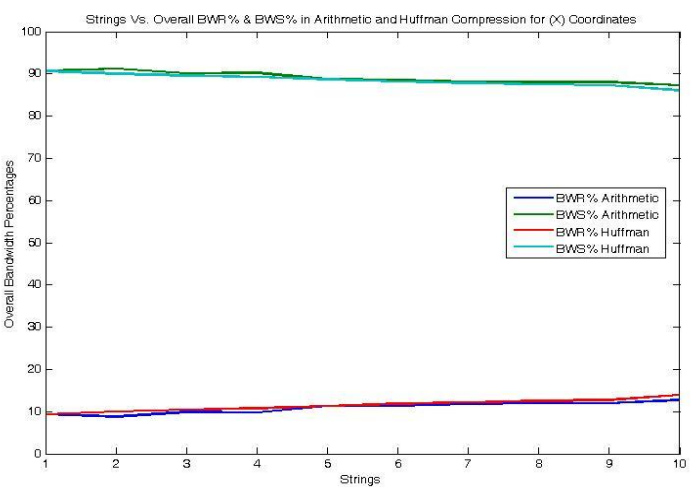

Figure 19: Overall BWR\% and BWS\%

From the above Table 28 and Figure 19, one can observe that, the overall percentage variation range in the bandwidth requirement and saving as follows:

\begin{tabular}{|c|c|c|c|}
\hline S.No. & Compression Type & BWR\% range & BWS\% range \\
\hline 1 & Arithmetic & $8.75-12.74$ & $87.25-91.25$ \\
\hline 2 & Huffman & $9.375-13.94$ & $86.05-90.625$ \\
\hline
\end{tabular}

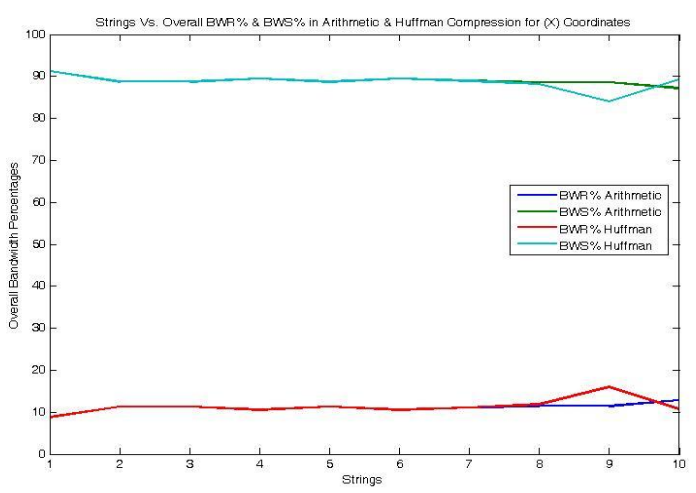

Figure 20: Overall BWR\% and BWS\%

Table 29 Overall BWR\% and BWS\% for case(i)

\begin{tabular}{|c|c|c|c|c|c|}
\hline \multirow{2}{*}{$\begin{array}{c}\text { Sl. } \\
\text { No. }\end{array}$} & \multirow{2}{*}{ Input String } & \multicolumn{2}{|c|}{ OBWR\% } & \multicolumn{2}{c|}{ OBWR\% } \\
\cline { 3 - 6 } & & Arithmetic & Huffman & Arithmetic & Huffman \\
\hline 1 & APPLE & 8.75 & 8.75 & 91.25 & 91.25 \\
\hline 2 & COMPUTER & 11.32813 & 11.32813 & 88.67188 & 88.67188 \\
\hline 3 & DOCUMENT & 11.32813 & 11.32813 & 88.67188 & 88.67188 \\
\hline 4 & ELEPHANT & 10.54688 & 10.54688 & 89.45313 & 89.45313 \\
\hline 5 & GRAPHICS & 11.32813 & 11.32813 & 88.67188 & 88.67188 \\
\hline 6 & HARDDISK & 10.54688 & 10.54688 & 89.45313 & 89.45313 \\
\hline 7 & BEAUTIFUL & 11.11111 & 11.11111 & 88.88889 & 88.88889 \\
\hline 8 & FLOWCHART & 11.45833 & 11.80556 & 88.54167 & 88.19444 \\
\hline 9 & JNTUCEVZM & 11.45833 & 15.97222 & 88.54167 & 84.02778 \\
\hline 10 & INFORMATION & 12.84722 & 10.7954 & 87.15278 & 89.2045 \\
\hline
\end{tabular}

From the above Table 29 and Figure 20, one can observe that, the overall percentage variation range in the bandwidth requirement and saving as follows: 


\begin{tabular}{|l|l|l|l|}
\hline S.No. & Compression Type & BWR\% range & BWS\% range \\
\hline 1 & Arithmetic & $8.75-12.84$ & $87.15-91.25$ \\
\hline 2 & Huffman & $8.75-15.97$ & $84.02-91.25$ \\
\hline
\end{tabular}

Table 30 Overall BWR\% and BWS\% for case(iii):

\begin{tabular}{|c|l|c|c|c|c|}
\hline \multirow{2}{*}{$\begin{array}{c}\text { Sl. } \\
\text { No. }\end{array}$} & \multirow{2}{*}{ Input String } & \multicolumn{2}{|c|}{ BWR\% } & \multicolumn{2}{c|}{ BWS\% } \\
\cline { 3 - 6 } & & Arithmetic & Huffman & Arithmetic & Huffman \\
\hline & AAAAAAAAAAAA & 7.102273 & 7.386364 & 92.89773 & 92.61364 \\
\hline 2 & AAAAAABBBBBB & 9.375 & 9.659091 & 90.625 & 90.34091 \\
\hline 3 & AAAABBBBCCCC & 7.386364 & 7.954545 & 92.61364 & 92.04545 \\
\hline 4 & AAABBBCCCDDD & 9.375 & 9.375 & 90.625 & 90.625 \\
\hline 5 & AABBCCDDEEFF & 9.090909 & 9.943182 & 90.90909 & 90.05682 \\
\hline 6 & AABBCCDDDEEE & 9.943182 & 10.51136 & 90.05682 & 89.48864 \\
\hline 7 & AAAABCCDDDD & 9.090909 & 9.659091 & 90.90909 & 90.34091 \\
\hline 8 & ABCDEEEFFFG & 5.681818 & 6.818182 & 94.31818 & 93.18182 \\
\hline 9 & ABCDEEEEEEF & 1.041667 & 3.385417 & 98.95833 & 96.61458 \\
\hline 10 & DDEEFGHHHII & 4.427083 & 5.208333 & 95.57292 & 94.79167 \\
\hline
\end{tabular}

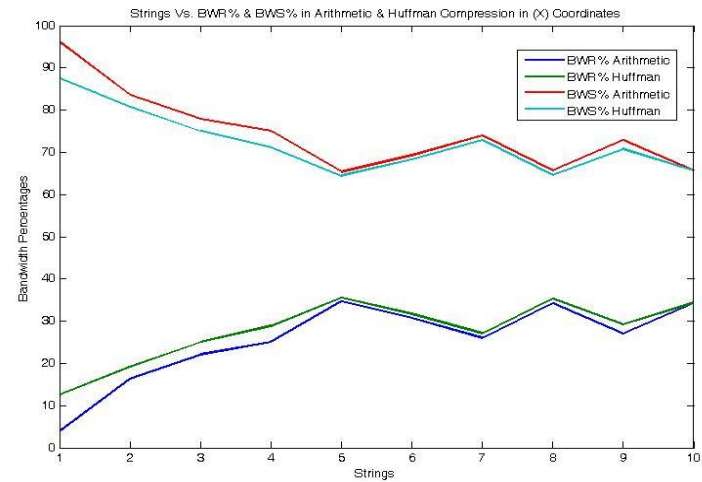

Figure 21: Overall BWR\% \& BWS\%

From the above Table 30 and Figure 21, one can observe that, the overall percentage variation range in the bandwidth requirement and saving as follows:

\begin{tabular}{|l|l|c|c|}
\hline S.No. & Compression Type & BWR\% range & BWS\% range \\
\hline 1 & Arithmetic & $1.04-9.94$ & $90.05-98.95$ \\
\hline 2 & Huffman & $1.38-10.51$ & $89.48-96.31$ \\
\hline
\end{tabular}

\section{Analysis of Overall BWR\% for Arithmetic and Huffman Compressions in $(X, Y)$ and $(X)$ Coordinates of Encrypted Data}

Table 31: Overall BWR\% for $(X, Y)$ Vs. $X$ co-ordinates in Huffman compression \& Arithmetic compression for Case (i)

\begin{tabular}{|c|c|c|c|c|c|}
\hline \multirow[b]{2}{*}{$\begin{array}{l}\text { SI. } \\
\text { No. }\end{array}$} & \multirow[b]{2}{*}{ Input String } & \multicolumn{2}{|c|}{ Huffman } & \multicolumn{2}{|c|}{ Arithmetic } \\
\hline & & $\begin{array}{c}\text { BWR\% } \\
(\mathbf{X}, \mathbf{Y})\end{array}$ & $\begin{array}{c}\text { BWR\% } \\
\text { (X) }\end{array}$ & $\begin{array}{c}\text { BWR\% } \\
(\mathbf{X}, \mathbf{Y})\end{array}$ & $\begin{array}{c}\text { BWR\% } \\
(\mathrm{X})\end{array}$ \\
\hline & $\mathrm{ABCD}$ & 26.5625 & 9.375 & 25.78125 & 9.375 \\
\hline 2 & ABCDE & 27.5 & 10 & 26.25 & 8.75 \\
\hline 3 & ABCDEF & 28.125 & 10.41667 & 27.60417 & 9.895833 \\
\hline 4 & ABCDEFG & 28.57143 & 10.71429 & 26.78571 & 9.821429 \\
\hline 5 & ABCDEFGH & 29.6875 & 11.32813 & 23.82813 & 11.32813 \\
\hline 6 & ABCDEFGHI & 30.55556 & 11.80556 & 21.18056 & 11.45833 \\
\hline 7 & ABCDEFGHIJ & 31.25 & 12.1875 & 19.0625 & 11.875 \\
\hline 8 & ABCDEFGHIJK & 31.81818 & 12.5 & 17.61364 & 11.93182 \\
\hline 9 & ABCDEFGHIJKL & 32.29167 & 12.76042 & 15.88542 & 11.97917 \\
\hline 10 & ABCDEFGHIJKLM & 32.69231 & 13.94231 & 14.90385 & 12.74038 \\
\hline
\end{tabular}

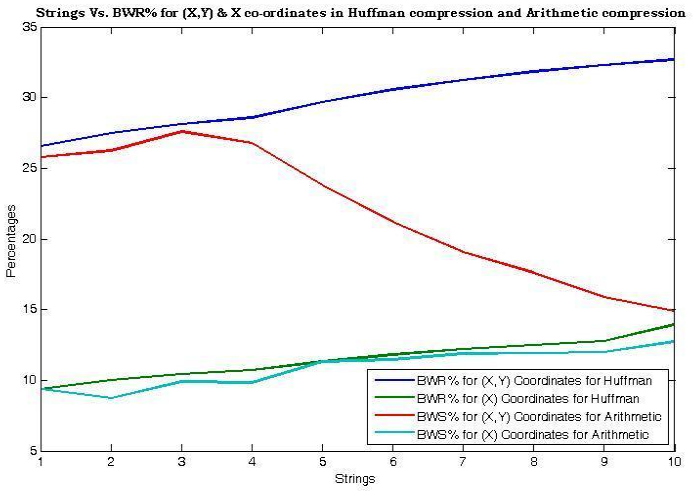

Figure 22: Strings Vs. BWR\% for $(X, Y) \&(X)$ co-ordinates in Huffman compression And Arithmetic compression

From the above Table 31 and Figure 22 one can observe the overall percentage of bandwidth requirement variation range in $(\mathrm{x}, \mathrm{y})$ and $\mathrm{x}$ co-ordinates as follows.

\begin{tabular}{|l|l|l|l|}
\hline S.No. & \multirow{2}{*}{ Compression } & \multicolumn{2}{|c|}{ BWR\% range } \\
\cline { 3 - 4 } & & \multicolumn{1}{|c|}{$(\mathrm{x}, \mathrm{v})$} & $\mathrm{x}$ \\
\hline 1 & Arithmetic & $14.92-27.6$ & $8.75-12.74$ \\
\hline 2 & Huffman & $26.56-32.69$ & $9.37-13.94$ \\
\hline
\end{tabular}

Table 32: Overall BWR\% for $(X, Y)$ Vs. $X$ co-ordinates in Huffman compression \& Arithmetic compression for Case (ii)

\begin{tabular}{|c|c|c|c|c|c|}
\hline \multirow[b]{2}{*}{$\begin{array}{l}\text { Sl. } \\
\text { No. }\end{array}$} & \multirow[b]{2}{*}{ Input String } & \multicolumn{2}{|c|}{ Huffman } & \multicolumn{2}{|c|}{ Arithmetic } \\
\hline & & $\begin{array}{l}\text { BWR\% } \\
(\mathbf{X}, \mathbf{Y})\end{array}$ & $\begin{array}{l}\text { BWR\% } \\
\text { (X) }\end{array}$ & $\begin{array}{l}\text { BWR\% } \\
(\mathbf{X}, \mathbf{Y})\end{array}$ & $\begin{array}{l}\text { BWR } \\
\% \\
(\mathbf{X})\end{array}$ \\
\hline 1 & APPLE & 25 & 8.75 & 24.375 & 8.75 \\
\hline 2 & COMPUTER & 29.687 & 11.328 & 23.828 & 11.328 \\
\hline 3 & DOCUMENT & 28.906 & 11.328 & 23.828 & 11.328 \\
\hline 4 & ELEPHANT & 28.125 & 10.546 & 23.046 & 10.546 \\
\hline
\end{tabular}




\begin{tabular}{|l|l|c|c|c|c|}
\hline 5 & GRAPHICS & 29.687 & 11.328 & 23.828 & 11.328 \\
\hline 6 & HARDDISK & 28.125 & 10.546 & 23.046 & 10.546 \\
\hline 7 & BEAUTIFUL & 29.166 & 11.111 & 21.180 & 11.111 \\
\hline 8 & FLOWCHART & 31.25 & 11.805 & 21.180 & 11.458 \\
\hline 9 & JNTUCEVZM & 29.861 & 15.972 & 21.180 & 11.458 \\
\hline 10 & INFORMATION & 28.409 & 10.795 & 16.761 & 12.847 \\
\hline
\end{tabular}

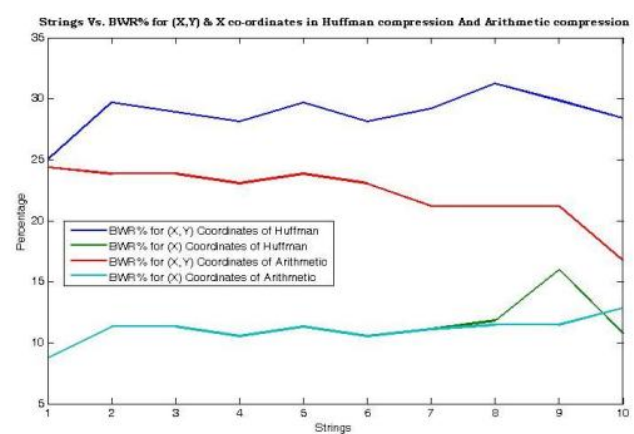

Figure 23: Strings Vs. BWR\% for $(\mathrm{X}, \mathrm{Y}) \&$ \& $(\mathrm{X})$ co-ordinates in Huffman compression And Arithmetic compression of overall

From the above Table 32 and Figure 23 one can observe the overall percentage of bandwidth requirement variation range in $(\mathrm{x}, \mathrm{y})$ and $\mathrm{x}$ co-ordinates as follows.

\begin{tabular}{|l|l|l|c|}
\hline S.No. & \multirow{2}{*}{ Compression } & \multicolumn{2}{|c|}{ BWR\% range } \\
\cline { 3 - 4 } & & $(\mathrm{x} . \mathrm{v})$ & $\mathrm{x}$ \\
\hline 1 & Arithmetic & $16.76-24.37$ & $8.75-12.84$ \\
\hline 2 & Huffman & $25-31.25$ & $8.75-15.97$ \\
\hline
\end{tabular}

Table 33: Overall BWR\% for $(X, Y)$ Vs. $X$ co-ordinates in Huffman compression And Arithmetic compression for Case (iii)

\begin{tabular}{|l|l|c|c|c|c|}
\hline \multirow{2}{*}{$\begin{array}{l}\text { SI. } \\
\text { No. }\end{array}$} & \multirow{2}{*}{ Input String } & \multicolumn{2}{|c|}{ Huffman } & \multicolumn{2}{c|}{ Arithmetic } \\
\cline { 3 - 6 } & & $\begin{array}{c}\text { BWR\% } \\
(\mathbf{X , Y})\end{array}$ & $\begin{array}{c}\text { BWR\% } \\
(\mathbf{X})\end{array}$ & $\begin{array}{c}\text { BWR\% } \\
(\mathbf{X , Y})\end{array}$ & $\begin{array}{c}\text { BWR\% } \\
(\mathbf{X})\end{array}$ \\
\hline & AAAABCCDDDD & 21.59091 & 7.386364 & 15.90909 & 7.102273 \\
\hline 2 & ABCDEEEFFFG & 26.13636 & 9.659091 & 17.32955 & 9.375 \\
\hline 3 & ABCDEEEEEEF & 22.72727 & 7.954545 & 17.32955 & 7.386364 \\
\hline 4 & DDEEFGHHHII & 25.56818 & 9.375 & 16.76136 & 9.375 \\
\hline 5 & KLKLFGHIJJ & 26.70455 & 9.943182 & 17.61364 & 9.090909 \\
\hline 6 & AABCCCDHIJ & 27.84091 & 10.51136 & 17.61364 & 9.943182 \\
\hline 7 & AAABBBCDEFJ & 26.13636 & 9.659091 & 16.76136 & 9.090909 \\
\hline 8 & AAAABBBBCCC & 19.88636 & 6.818182 & 17.04545 & 5.681818 \\
\hline 9 & AAAAAAAAAAAA & 10.9375 & 3.385417 & 9.375 & 1.041667 \\
\hline 10 & AAAAAAABBBBBB & 16.14583 & 5.208333 & 15.625 & 4.427083 \\
\hline
\end{tabular}

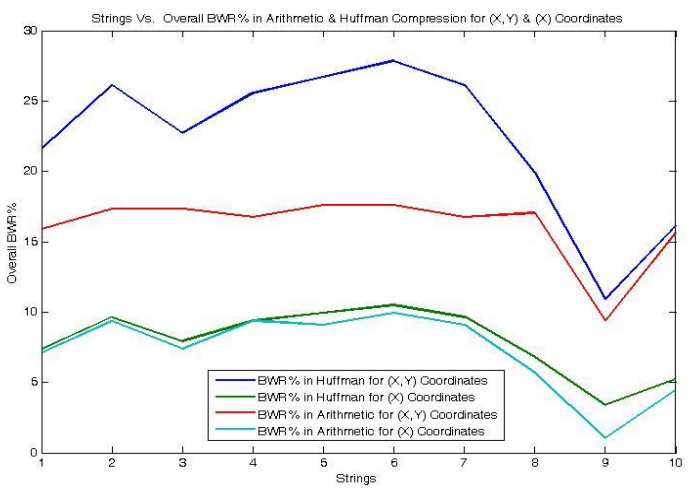

Figure 24: Strings Vs. BWR\% for $(X, Y) \&(X)$ co-ordinates in Huffman compression And Arithmetic compression

From the above Table 33 and Figure 24 one can observe the overall percentage of bandwidth requirement variation range in $(\mathrm{x}, \mathrm{y})$ and $\mathrm{x}$ co-ordinates as follows.

\begin{tabular}{|l|l|c|c|}
\hline S.No. & \multirow{2}{*}{ Compression } & \multicolumn{2}{|c|}{ BWR\% range } \\
\cline { 3 - 4 } & & $(\mathrm{x}, \mathrm{v})$ & $\mathrm{x}$ \\
\hline 1 & Arithmetic & $9.375-17.61$ & $1.04-9.94$ \\
\hline 2 & Huffman & $10.93-27.84$ & $3.38-10.5$ \\
\hline
\end{tabular}

\section{ANALYSIS OF OVERALL BWS\% FOR ARITHMETIC AND HUFFMAN COMPRESSIONS IN (X,Y) AND (X) COORDINATES OF ENCRYPTED DATA}

Table 34: Overall BWS\% for $(X, Y)$ Vs. $X$ co-ordinates in Huffman compression \& Arithmetic compression for Case (i)

\begin{tabular}{|l|l|l|l|l|l|}
\hline \multirow{2}{*}{$\begin{array}{l}\text { Sl. } \\
\text { No. }\end{array}$} & \multirow{2}{*}{ Input String } & \multicolumn{2}{|c|}{ Huffman } & \multicolumn{2}{|c|}{ Arithmetic } \\
\cline { 3 - 6 } & & $\begin{array}{l}\text { BWS\% } \\
(\mathbf{X , Y})\end{array}$ & $\begin{array}{l}\text { BWS\% } \\
(\mathbf{X})\end{array}$ & $\begin{array}{l}\text { BWS\% } \\
(\mathbf{X , Y})\end{array}$ & $\begin{array}{l}\text { BWS\% } \\
(\mathbf{X})\end{array}$ \\
\hline 1 & ABCD & 73.4375 & 90.625 & 74.21875 & 90.625 \\
\hline 2 & ABCDE & 72.5 & 90 & 73.75 & 91.25 \\
\hline 3 & ABCDEF & 71.875 & 89.58333 & 72.39583 & 90.10417 \\
\hline 4 & ABCDEFG & 71.42857 & 89.28571 & 73.21429 & 90.17857 \\
\hline 5 & ABCDEFGH & 70.3125 & 88.67188 & 76.17188 & 88.67188 \\
\hline 6 & ABCDEFGHI & 69.44444 & 88.19444 & 78.81944 & 88.54167 \\
\hline 7 & ABCDEFGHIJ & 68.75 & 87.8125 & 80.9375 & 88.125 \\
\hline 8 & ABCDEFGHIJ & 68.18182 & 87.5 & 82.38636 & 88.06818 \\
\hline 9 & ABCDEFGHIJKL & 67.70833 & 87.23958 & 84.11458 & 88.02083 \\
\hline 10 & ABCDEFGHIJKLM & 67.30769 & 86.05769 & 85.09615 & 87.25962 \\
\hline
\end{tabular}




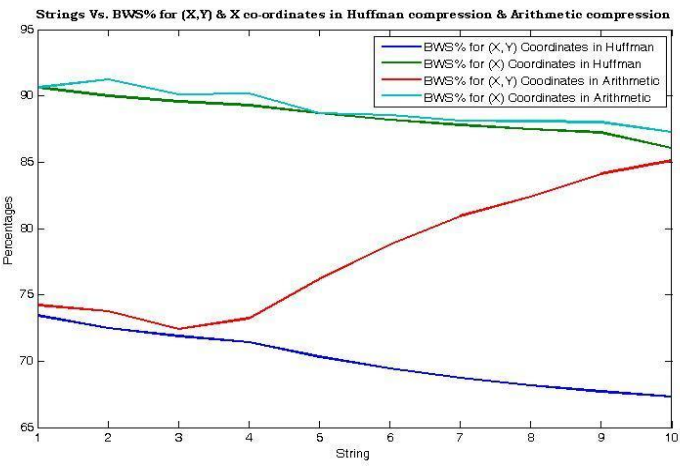

Figure 25: Strings Vs. BWS \% for $(X, Y) \& X$ co-ordinates in Huffman compression And Arithmetic compression

From the above Table 34 and Figure 25 one can observe that, the overall percentage of bandwidth saving variation range for $(\mathrm{x}, \mathrm{y})$ and $\mathrm{x}$ co-ordinates as follows.

\begin{tabular}{|l|l|c|c|}
\hline S.No. & \multirow{2}{*}{ Compression } & \multicolumn{2}{|c|}{ BWS\% range } \\
\cline { 3 - 4 } & & $(\mathrm{x} . \mathrm{v})$ & $\mathrm{x}$ \\
\hline 1 & Arithmetic & $72.39-85.09$ & $87.25-91.25$ \\
\hline 2 & Huffman & $67.3-73.43$ & $86.05-90.625$ \\
\hline
\end{tabular}

Table 36: Overall BWS\% for $(X, Y)$ Vs. $X$ co-ordinates in Huffman compression And Arithmetic compression for Case (ii)

\begin{tabular}{|l|l|c|l|l|c|}
\hline \multirow{2}{*}{$\begin{array}{l}\text { Sl. } \\
\text { No. }\end{array}$} & \multirow{2}{*}{ Input String } & \multicolumn{2}{|c|}{ Huffman } & \multicolumn{2}{c|}{ Arithmetic } \\
\cline { 3 - 6 } & & $\begin{array}{l}\text { BWS\% } \\
(\mathbf{X}, \mathbf{Y})\end{array}$ & $\begin{array}{l}\text { BWS\% } \\
(\mathbf{X})\end{array}$ & $\begin{array}{l}\text { BWS\% } \\
(\mathbf{X}, \mathbf{Y})\end{array}$ & $\begin{array}{l}\text { BWS\% } \\
(\mathbf{X})\end{array}$ \\
\hline & APPLE & 75 & 91.25 & 75.625 & 91.25 \\
\hline 2 & COMPUTER & 70.3125 & 88.67188 & 76.17188 & 88.67188 \\
\hline 3 & DOCUMENT & 71.09375 & 88.67188 & 76.17188 & 88.67188 \\
\hline 4 & ELEPHANT & 71.875 & 89.45313 & 76.95313 & 89.45313 \\
\hline 5 & GRAPHICS & 70.3125 & 88.67188 & 76.17188 & 88.67188 \\
\hline 6 & HARDDISK & 71.875 & 89.45313 & 76.95313 & 89.45313 \\
\hline 7 & BEAUTIFUL & 70.83333 & 88.88889 & 78.81944 & 88.88889 \\
\hline 8 & FLOWCHART & 68.75 & 88.19444 & 78.81944 & 88.54167 \\
\hline 9 & JNTUCEVZM & 70.13889 & 84.02778 & 78.81944 & 88.54167 \\
\hline 10 & INFORMATION & 71.59091 & 89.2045 & 83.23864 & 87.15278 \\
\hline
\end{tabular}

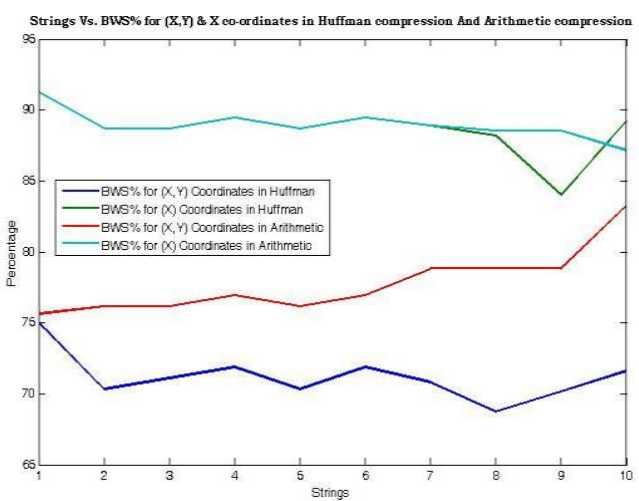

Figure 26: Strings Vs. BWS \% for $(X, Y) \&(X)$ co-ordinates in Huffman compression And Arithmetic compression
From the above Table 36 and Figure 26 one can observe that, the overall percentage of bandwidth saving variation range for $(\mathrm{x}, \mathrm{y})$ and $\mathrm{x}$ co-ordinates as follows.

\begin{tabular}{|l|l|l|c|}
\hline S.No. & \multirow{2}{*}{ Compression } & \multicolumn{2}{|c|}{ BWS\% range } \\
\cline { 3 - 4 } & & \multicolumn{1}{|c|}{$(\mathrm{x}, \mathrm{y})$} & $\mathrm{x}$ \\
\hline 1 & Arithmetic & $75.625-83.23$ & $87.15-91.25$ \\
\hline 2 & Huffman & $68.75-75$ & $84.04-91.25$ \\
\hline
\end{tabular}

Table 36: Overall BWS\% for $(X, Y)$ Vs. $X$ co-ordinates in Huffman compression And Arithmetic compression Case (iii)

\begin{tabular}{|l|l|l|l|l|c|}
\hline \multirow{2}{*}{$\begin{array}{l}\text { SI. } \\
\text { No. }\end{array}$} & \multirow{2}{*}{ Input String } & \multicolumn{2}{|c|}{ Huffman } & \multicolumn{2}{c|}{ Arithmetic } \\
\cline { 3 - 6 } & & $\begin{array}{l}\text { BWS\% } \\
(\mathbf{X , Y})\end{array}$ & $\begin{array}{l}\text { BWS\% } \\
(\mathbf{X})\end{array}$ & $\begin{array}{l}\text { BWS\% } \\
(\mathbf{X , Y})\end{array}$ & $\begin{array}{l}\text { BWS\% } \\
(\mathbf{X})\end{array}$ \\
\hline & AAAABCCDDDD & 78.40909 & 92.61364 & 84.09091 & 92.89773 \\
\hline 2 & ABCDEEEFFFG & 73.86364 & 90.34091 & 82.67045 & 90.625 \\
\hline 3 & ABCDEEEEEEF & 77.27273 & 92.04545 & 82.67045 & 92.61364 \\
\hline 4 & DDEEFGHHHII & 74.43182 & 90.625 & 83.23864 & 90.625 \\
\hline 5 & KLKLFGHIJJ & 73.29545 & 90.05682 & 82.38636 & 90.90909 \\
\hline 6 & AABCCCDHIJ & 72.15909 & 89.48864 & 82.38636 & 90.05682 \\
\hline 7 & AAABBBCDEFJ & 73.86364 & 90.34091 & 83.23864 & 90.90909 \\
\hline 8 & AAAABBBBCCC & 80.11364 & 93.18182 & 82.95455 & 94.31818 \\
\hline 9 & AAAAAAAAAAAA & 89.0625 & 96.61458 & 90.625 & 98.95833 \\
\hline 10 & AAAAAABBBBBB & 83.85417 & 94.79167 & 84.375 & 95.57292 \\
\hline
\end{tabular}

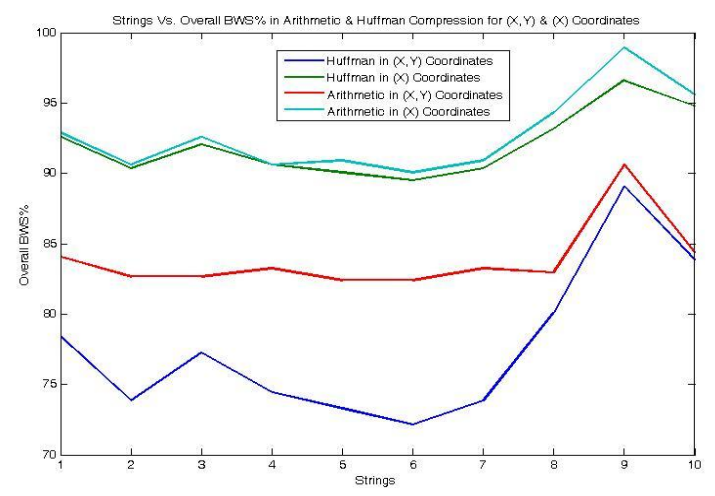

Figure 27: Strings Vs. BWS \% for (X,Y) Vs. (X) co-ordinates in Huffman compression And Arithmetic compression

From the above Table 36 and Figure 27 one can observe that, the overall percentage of bandwidth saving variation range for $(\mathrm{x}, \mathrm{y})$ and $\mathrm{x}$ co-ordinates as follows.

\begin{tabular}{|l|l|l|l|}
\hline S.No. & \multirow{2}{*}{ Compression } & \multicolumn{2}{|c|}{ BWS\% range } \\
\cline { 3 - 4 } & & $(\mathrm{x}, \mathrm{v})$ & $\mathrm{x}$ \\
\hline 1 & Arithmetic & $72.15-89.06$ & $89.48-96.61$ \\
\hline 2 & Huffman & $82.67-91.625$ & $90.05-98.95$ \\
\hline
\end{tabular}




\section{CONCLUSION}

We have conducted the experiments for the following cases, by considering only $x$ co-ordinate and both $(x, y)$ co-ordinates of the different encrypted text for transmission in Arithmetic as well as in Huffman for small text and as the Arithmetic compression is not suitable for large text, we consider only Huffman compression :

For small text, irrespective of the case, when we consider both $(\mathrm{x}, \mathrm{y})$ coordinates for transmission, the overall Percentage of bandwidth requirement varies from $10.93 \%$ to $32.69 \%$ and the percentage of Bandwidth Saving (BWS) varies from $67.3 \%$ to $89.06 \%$ in Huffman whereas the overall percentage of bandwidth requirement varies from $9.375 \%$ to $27.6 \%$ and the percentage of Bandwidth Saving (BWS) varies from $72.39 \%$ to 90.625\% in Arithmetic.

When we consider only $x$ co-ordinate for transmission, the overall percentage of bandwidth requirement varies from $3.38 \%$ to $15.97 \%$ and the percentage of Bandwidth Saving (BWS) varies from $84.02 \%$ to $96.61 \%$ in Huffman, whereas the overall percentage of bandwidth requirement varies from $1.04 \%$ to $12.84 \%$ and the percentage of Bandwidth Saving (BWS) varies from $87.15 \%$ to $98.95 \%$, in Arithmetic.

Hence, from the above experimental observations, we conclude that, irrespective of the data, Arithmetic Compression is more suitable for small text when compared with Huffman compression and for large text Huffman compression is suitable.

\section{REFERENCES}

[1] Neal Koblitz, "Elliptic Curve Cryptosystem, Journal of mathematics computation Vol.48, No.177pp.203-209,Jan1987.

[2] V. Miller, "Uses of elliptic curves in cryptography", Advances in Cryptology-Crypto '85,Lecture Notes in Computer Science, 218

[3] Certicom Corp., " An Introduction to Information Security", Number 1, March 1997.

[4] ANSI X9.63, Public Key Cryptography for the Financial Services Industry: Elliptic CurveKey Agreement and Key Transport Protocols, ballot version, May 2001.

[5] Internet Engineering Task Force, The OAKLEY Key Determination Protocol, IETF RFC 2412, November 1998.

[6] ISO/IEC 15946-3, Information Technology-Security Techniques-Cryptographic TechniquesBased on Elliptic Curves, Part 3, Final Draft International Standard (FDIS), February 2001

[7] National Institute of Standards and Technology, Digital Signature Standard, FIPS Publication186-2, 2000.

[8] M. Jacobson, N. Koblitz, J. Silverman, A. Stein and E. Teske, "Analysis of the xedni calculus attack", Designs, Codes and Cryptography, 20 (2000), 41-64. (1986), Springer-Verlag, 417-426.

[9] Standards for Efficient Cryptography Group, SEC 1: Elliptic Curve Cryptography, version1.0, 2000. Available at http://www.secg.org
[10] R.L. Rivest, A. Shamir, and L.M. Adleman, Method for Obtaining Digital Signatures and Public-key Cryptosystems", Communications of the ACM,Volume 21, pages 120-126, February 1978.

[11] S. Arita, "Weil descent of elliptic curves over finite fields of characteristic three", Advances in Cryptology-Asiacrypt 2000, LectureNotes in Computer Science, 1976 (2000),Springer-Verlag, 248-259

[12] Fernandes, A. "Elliptic Curve Cryptography”, Dr.Dobb's journal, December 1999

[13] D. A. Huffman, "A method for the construction of minimum redundancy codes", Proc. IRE, Vol. 40, No. 9, pp. 1098-1101, September 1952.

[14 Rissanen, J.J. Generalized Kraft inequality and arithmetic coding.IBM 1. Res. Dev. 20 (May 1976), 198-203. Another early exposition of the idea of arithmetic coding.

[15] Pasco, R. (1976) "Source Coding Algorithms for Fast Data Compression," Ph. D. dissertation, Dept. of Electrical Engineering, Stanford University, Stanford, Calif

[16] Rissanen. J.J. Arithmetic codings as number representations. Acta Polytech. Stand. Math. 31 (Dec. 1979), 44-51. Further develops arithmetic coding as a practical technique for data representation.

[17] Rissanen, J., and Langdon, G.G. Arithmetic coding. IBM J. Res. Dev.23, 2 (Mar. 1979). 149-162. Describes a broad class of arithmetic codes.

[18] Langdon, G.G. An introduction to arithmetic coding. IBMI. Res. Dev. 28, 2 (Mar. 1984), 135-149. Introduction to arithmetic coding from the point of view of hardware implementation.

[19] A.Moffat and J.Katajainen, "In-place calculation of minimum-redundancy codes", 4th Intl. Workshop on Algorithms and Data Structures, Vol. 955, pp. 393-402, August 1995.

[20] J.Van Leeuwen, "On the construction of Huffman trees", 3rd International Colloquium on Automata, Languages and Programming, pp. 382-410, July 1976.

[21] M. Buro, "On the maximum length of Huffman codes", Information Processing Letters, Vol. 45, No.5, pp. 219-223, April 1993.

[22] H. C. Chen, Y. L. Wang and Y. F. Lan, "A memory efficient and fast Huffman decoding algorithm", Information Processing Letters, Vol. 69, No. 3, pp. 119122, February 1999.

[23] R. Hashemian, "Direct Huffman coding and decoding using the table of code-lengths", Proc. International Conf. on Inform. Technology: Computers and Communications (ITCC '03), pp. 237-241, April 2003.

[24] S. Ho and P. Law, "Efficient hardware decoding method for modified Huffman code", Electronics Letters, Vol. 27, No. 10, pp. 855-856, May 1991. 
[25] S. T. Klein, "Skeleton trees for the efficient decoding of Huffman encoded texts", Kluwer Journal of Inform. Retrieval, Vol. 3, No. 1, pp. 7-23, July 2000.

[26] L. L. Larmore and D. S. Hirschberg, "A fast algorithm for optimal length-limited Huffman codes", Journal of ACM, Vol. 37, No. 3, pp. 464-473, July 1999.

[27] A. Moffat and A. Turpin, "On the implementation of minimum-redundancy prefix codes", IEEE Trans. Commun., Vol. 45, No. 10, pp. 1200-1207, October 1997.

[28] O.Srinivasa Rao, S.Pallam Setty, "Efficient mapping methods of Elliptic Curve Crypto Systems" International Journal of Engineering Science and Technology, Vol. 2(8), 2010, pp. 3651-3656

[29] Vigila, S.; Muneeswaran, K.; "Implementation of text based cryptosystem using Elliptic Curve Cryptography", Advanced Computing, 2009. ICAC 2009. First
International Conference on 13-15 Dec. 2009, Onpage(s): $82-85$.

[30] Gupta, K.; Silakari, S.; Gupta, R.; Khan, S.A.; “An Ethical Way of Image Encryption Using ECC" Computational Intelligence, Communication Systems and Networks, 2009. CICSYN '09. First International Conference on 23-25 July 2009, Onpage(s):342-345.

[31] R. Rajaram Ramasamy, M. Amutha Prabakar, M. Indra Devi, and M. Suguna, "Knapsack Based ECC Encryption and Decryption" International Journal of Network Security, Vol.9, No.3, PP.218-226, Nov. 2009

[32] O.Srinivasa Rao, S.Pallam Setty, "Comparative Study of Arithmetic and Huffman Data Compression Techniques for Koblitz Curve Cryptography" International Journal of Computer Applications (0975 - 8887),Volume 14- No.5, January 2011 